\title{
ON A CERTIFIED SMAGORINSKY REDUCED BASIS TURBULENCE MODEL*
}

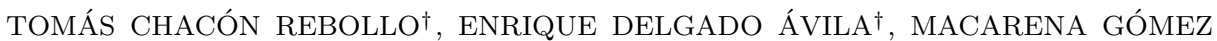 \\ MÁRMOL ${ }^{\ddagger}$, FRANCESCO BALLARIN ${ }^{\S}$, AND GIANLUIGI ROZZA ${ }^{\S}$
}

\begin{abstract}
In this work we present a reduced basis Smagorinsky turbulence model for steady flows. We approximate the nonlinear eddy diffusion term using the empirical interpolation method (cf. [M. A. Grepl et al., ESAIM Math. Model. Numer. Anal., 41 (2007), pp. 575-605; Barrault et al., C. R. Acad. Sci. Paris Sér. I Math., 339 (2004), pp. 667-672]) and the velocity-pressure unknowns by an independent reduced-basis procedure. This model is based upon an a posteriori error estimation for a Smagorinsky turbulence model. The theoretical development of the a posteriori error estimation is based on [S. Deparis, SIAM J. Sci. Comput., 46 (2008), pp. 2039-2067] and [A. Manzoni, ESAIM Math. Model. Numer. Anal., 48 (2014), pp. 1199-1226], according to the Brezzi-Rappaz-Raviart stability theory, and adapted for the nonlinear eddy diffusion term. We present some numerical tests, programmed in FreeFem++ (cf. [F. Hecht, J. Numer. Math., 20 (2012), pp. 251-265]), in which we show a speedup on the computation by factor larger than 1000 in benchmark two-dimensional flows.
\end{abstract}

Key words. reduced basis method, empirical interpolation method, a posteriori error estimation, steady Smagorinsky model

AMS subject classifications. 65N12, 65N15, 65N30, 76F 65

DOI. $10.1137 / 17 \mathrm{M} 1118233$

1. Introduction. Reduced order modeling (ROM) has been successfully used in several fields to provide a large reduction in computation cost for the solution of partial differential equations $[19,20,27,28,29,30]$. In fluid mechanics a popular strategy is to use proper orthogonal decomposition (POD) to extract the dominant structures for high-Reynolds flow, which are then used in a Galerkin approximation of the underlying equations $[20,35]$. There have been a number of recent works combining $\mathrm{POD} /$ reduced basis (RB) with variational multiscale models [39], ensemble models [16], flow regularization models [2, 4], as well as bifurcation problems [18, 25, 26, 40] all in the framework of the incompressible Navier-Stokes equations. Application of the POD-Galerkin strategy to turbulent fluid flows remains a challenging area of research. By construction, ROMs generated using only the first most energetic POD basis functions are not endowed with the dissipative mechanisms associated to the creation of lower size, and less energetic, turbulent scales. Increasing the number of modes creates very large POD-Galerkin ROMs that are still very computationally expensive to solve (cf. [3] and references therein).

A developing way of research to overcome this difficulty is to adapt the standard turbulent closure techniques based upon eddy dissipation to model the effect of the unresolved ROM modes on the resolved ones. This is based upon the analysis of

\footnotetext{
*Received by the editors March 7, 2017; accepted for publication (in revised form) August 23, 2017; published electronically December 7, 2017.

http://www.siam.org/journals/sinum/55-6/M111823.html

Funding: This work has been supported by Spanish Government Project MTM2015-64577-C21-R and COST Action TD1307.

†IMUS \& Departamento de Ecuaciones Diferenciales y Análisis Numérico, Universidad de Sevilla, 41080 Seville, Spain (chacon@us.es, edelgado1@us.es).

${ }_{\ddagger}^{\ddagger}$ Departamento de Ecuaciones Diferenciales y Análisis Numérico, Universidad de Sevilla, 41080 Seville, Spain (macarena@us.es).

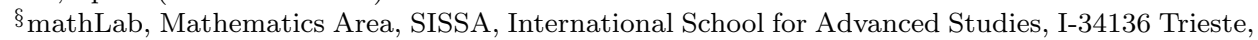
Italy (francesco.ballarin@sissa.it, gianluigi.rozza@sissa.it).
} 
[12], which shows that the transfer of energy among the POD modes is similar to the transfer of energy among Fourier modes; in fact there is a net energy transfer from low index POD modes to higher index POD modes (cf. [38] and references therein).

In this paper we address an alternative strategy that consists of constructing ROMs of turbulence models, rather than using ROM to construct turbulence models. We assume that the flow under consideration (or, rather, its large scales) is well modeled by the starting turbulence model, at least up to the accuracy required by the targeted application. Our purpose is to construct fast solvers for the turbulence model which is a highly nonlinear mathematical system of equations (often with larger and more complex nonlinearities than the Navier-Stokes equations) and needs large computational times to be solved. Current engineering applications for design, optimization, and control require repeated queries to turbulent models, so there is a heavy interest in following this approach.

We address the systematic construction of an RB Smagorinsky turbulence model (cf. [36]), which is the basic large eddy simulation (LES) turbulence model, in which the effect of the subgrid scales on the resolved scales is modeled by eddy diffusion terms (cf. $[11,34])$. It is an intrinsically discrete model, since the eddy viscosity term depends on the mesh size.

RB methods for incompressible fluid flows were first introduced for the Stokes equations (see, e.g., $[24,32,31]$ ). The RB is constructed by means of greedy algorithms, an a posteriori error bound estimator based upon the dual norm of the residual, and the inf-sup constant developed for the Stokes problem. This idea was then extended for the Navier-Stokes problem (see, e.g., [13, 14, 22, 37]), developing the a posteriori error estimator taking into account the Brezzi-Rappaz-Raviart (BRR) theory (cf. [8]). We extend in this paper the approach for the reduction of Navier-Stokes equations to the Smagorinsky turbulence model.

Unlike the Navier-Stokes problem that has an affine formulation with respect to the physical parameters, the Smagorinsky eddy viscosity term leads to a nonaffine formulation with respect to the parameter for the Smagorinsky model. We use the empirical interpolation method (EIM), introduced in [15] and [5], to approximate the nonlinear eddy viscosity term of the Smagorinsky model, obtaining an affine formulation with respect to the parameter. Thanks to this technique, we can store in the offline phase parameter-independent matrices, obtaining in the online phase a fast and highly accurate computation of the eddy diffusion term.

The construction of the reduced spaces for velocity and pressure is made by means of a greedy algorithm. To reduce the time to compute the errors within this algorithm, we have developed an a posteriori error bound estimator based on the BRR theory (see [8]). The use of a norm that takes into account the eddy viscosity effects allows a better initialization of the greedy algorithm.

We have performed several tests of the reduced model to solve the two-dimensional (2D) step and cavity flows, with the Reynolds number ranging in intervals in which a steady solution is known to exist. We obtain speed-up rates of several order of magnitude, where errors are normalized with respect to the finite element solution below $10^{-4}$.

The structure of this paper is as follows. In section 2, we present the continuous and discrete problems we work with. The RB method is considered in 3, where we describe the greedy algorithm that we use to select the different snapshots. After that, in section 4, we present the numerical analysis that we need in order to ensure the wellpossedness, based on the BRR theory, of the discrete problem presented in section 2 . The construction and analysis of the a posteriori error bound estimator is presented 
in section 5 . Then, in section 6 , we explain more in detail how we treat the nonlinear eddy viscosity corresponding with the Smagorinsky term. Finally, we present some numerical results in section 7 , where we show the reduction of the computational time in two different tests.

2. Finite element Smagorinsky model. To formulate the Smagorinsky turbulence model, let $\Omega$ be a bounded polyhedral domain in $\mathbb{R}^{d},(d=2,3)$. We assume that its boundary is split into $\Gamma=\Gamma_{D} \cup \Gamma_{N}$, where $\Gamma_{D}=\Gamma_{D_{g}} \cup \Gamma_{D_{0}}$ is the boundary relative to the nonhomogeneous and homogeneous Dirichlet boundary conditions, and $\Gamma_{N}$ to the Neumann conditions.

Let $\left\{\mathcal{T}_{h}\right\}_{h>0}$ a family of affine-equivalent and conforming triangulations of $\bar{\Omega}$, formed by triangles or quadrilaterals $(d=2)$, tetrahedra or hexaedra $(d=3)$. As usual the parameter $h$ is the maximum diameter $h_{K}$ among the elements $K \in \mathcal{T}_{h}$.

Although the Smagorinsky model is intrinsically discrete, it can be interpreted as a discretization of a continuous model. We next present this model to clarify its relationship with the Navier-Stokes equations. In this way, the "continuous" Smagorinsky turbulence model is formulated as

$$
\begin{cases}\mathbf{w} \cdot \nabla \mathbf{w}+\nabla p-\nabla \cdot\left(\left(\frac{1}{\mu}+\nu_{T}(\mathbf{w})\right) \nabla \mathbf{w}\right)=\mathbf{f} & \text { in } \Omega, \\ \nabla \cdot \mathbf{w}=0 & \text { in } \Omega, \\ \mathbf{w}=\mathbf{g}_{D} & \text { on } \Gamma_{D_{g}}, \\ \mathbf{w}=0 & \text { on } \Gamma_{D_{0}}, \\ -p \mathbf{n}+\left(\frac{1}{\mu}+\nu_{T}(\mathbf{w})\right) \frac{\partial \mathbf{w}}{\partial \mathbf{n}}=0 & \text { on } \Gamma_{N},\end{cases}
$$

where $\mu$ is the Reynolds number, $\mathbf{w}=\mathbf{w}(\mu)$ is the velocity field, and $p=p(\mu)$ is the pressure, both depending on the Reynolds number.

The eddy diffusion term is given by $\nu_{T}(\mathbf{w})=C_{S}^{2} \sum_{K \in \mathcal{T}_{h}} h_{K}^{2}\left|\nabla \mathbf{w}_{\left.\right|_{K}}\right| \chi_{K}$, where $|\cdot|$ denotes the Frobenius norm in $\mathbb{R}^{d \times d}$, and $C_{S}$ is the Smagorinsky constant [33].

Let us consider the spaces $Y=\left\{\mathbf{v} \in H^{1}(\Omega):\left.\mathbf{v}\right|_{\Gamma_{D}}=0\right\}$ for velocity and $M=$ $L^{2}(\Omega)$ for pressure. We assume that there exists a lift function $\mathbf{u}_{D} \in\left(H^{1}(\Omega)\right)^{d}$, such that $\left.\mathbf{u}_{D}\right|_{\Gamma_{D_{g}}}=\mathbf{g}_{D},\left.\mathbf{u}_{D}\right|_{\Gamma_{D_{0}}}=0$, and $\nabla \cdot \mathbf{u}_{D}=0$ in $\Omega$. With those conditions, we ensure that the lifted velocity $\mathbf{u}=\mathbf{w}-\mathbf{u}_{D}$ is still incompressible and satisfies the homogeneous Dirichlet boundary conditions on $\Gamma_{D}$. We will assume that $\mathbf{f} \in\left(L^{2}(\Omega)\right)^{d}$ and $\mathbf{g}_{D} \in\left(H^{1 / 2}(\Omega)\right)^{d}$.

Let $Y_{h} \subset Y$ and $M_{h} \subset M$ be two finite subspaces of $Y$ and $M$. We consider the following variational discretization of problem (1), actually, the "true" Smagorinsky model:

$$
\left\{\begin{array}{l}
\text { Find }\left(\mathbf{u}_{h}, p_{h}\right)=\left(\mathbf{u}_{h}(\mu), p_{h}(\mu)\right) \in Y_{h} \times M_{h} \text { such that } \forall \mathbf{v}_{h} \in Y_{h}, \forall q_{h} \in M_{h} \\
a\left(\mathbf{u}_{h}, \mathbf{v}_{h} ; \mu\right)+b\left(\mathbf{v}_{h}, p_{h} ; \mu\right)+a_{S}\left(\mathbf{w}_{h} ; \mathbf{w}_{h}, \mathbf{v}_{h} ; \mu\right) \\
+c\left(\mathbf{u}_{h}, \mathbf{u}_{h}, \mathbf{v}_{h} ; \mu\right)+c\left(\mathbf{u}_{D}, \mathbf{u}_{h}, \mathbf{v}_{h} ; \mu\right)+c\left(\mathbf{u}_{h}, \mathbf{u}_{D}, \mathbf{v}_{h} ; \mu\right)=F\left(\mathbf{v}_{h} ; \mu\right), \\
b\left(\mathbf{u}_{h}, q_{h} ; \mu\right)=0,
\end{array}\right.
$$

where $\mathbf{w}_{h}=\mathbf{u}_{h}+\mathbf{u}_{D} ;$ the bilinear forms $a(\cdot, \cdot ; \mu)$ and $b(\cdot, \cdot ; \mu)$ are defined by

$$
a(\mathbf{u}, \mathbf{v} ; \mu)=\frac{1}{\mu} \int_{\Omega} \nabla \mathbf{u}: \nabla \mathbf{v} d \Omega, \quad b(\mathbf{v}, q ; \mu)=-\int_{\Omega}(\nabla \cdot \mathbf{v}) q d \Omega ;
$$

the trilinear form, $c(\cdot, \cdot, \cdot ; \mu)$, and the nonlinear Smagorinsky term, $a_{S}(\cdot ; \cdot, \cdot ; \mu)$, are 
given by

$$
c(\mathbf{z}, \mathbf{u}, \mathbf{v} ; \mu)=\int_{\Omega}(\mathbf{z} \cdot \nabla \mathbf{u}) \mathbf{v} d \Omega, \quad a_{S}(\mathbf{z} ; \mathbf{u}, \mathbf{v} ; \mu)=\int_{\Omega} \nu_{T}(\mathbf{z}) \nabla \mathbf{u}: \nabla \mathbf{v} d \Omega .
$$

Finally, the linear form $F(\cdot ; \mu)$ is defined by

$$
F(\mathbf{v} ; \mu)=\langle\mathbf{f}, \mathbf{v}\rangle-a\left(\mathbf{u}_{D}, \mathbf{v} ; \mu\right)-c\left(\mathbf{u}_{D}, \mathbf{u}_{D}, \mathbf{v} ; \mu\right),
$$

where $\langle\cdot, \cdot\rangle$ stands for the duality pairing between $Y^{\prime}$ and $Y, Y^{\prime}$ being the dual space of $Y$.

The solution of problem (2) is intended to approximate the large-scale component of the solution of the Navier-Stokes problem (i.e., problem (1) with $\nu_{T}=0$ ).

Let us define the norms relative to the spaces $Y$ and $M$. For the velocity space $Y$, we consider a weighted inner product, $(\cdot, \cdot)_{T}$, defined as

$$
(\mathbf{u}, \mathbf{v})_{T}=\int_{\Omega}\left[\frac{1}{\bar{\mu}}+\nu_{T}^{*}\right] \nabla \mathbf{u}: \nabla \mathbf{v} d \Omega \quad \forall \mathbf{u}, \mathbf{v} \in Y
$$

where $\nu_{T}^{*}=\nu_{T}(\mathbf{w}(\bar{\mu})), \bar{\mu}=\arg \min _{\mu \in \mathcal{D}}\left\{\sum_{K \in \mathcal{T}_{h}}\left(C_{S} h_{K}\right)^{2} \min _{x \in K}|\nabla \mathbf{w}(\mu)|(x) \chi_{K}(x)\right\}$, and $\mathbf{w}(\mu)$ is the velocity solution of (1). This inner product induces a norm linked to the eddy diffusion term, $\|\cdot\|_{T}=(\cdot, \cdot)_{T}^{1 / 2}$. As the functions of $Y$ vanish on $\Gamma_{D}$, then, this norm is equivalent to the usual $H^{1}$ norm. This norm will turn out to be crucial to apply our error estimator in the RB construction by the greedy algorithm. For the pressure space $M$, we will use the usual $L^{2}$-norm, denoted by $\|\cdot\|_{0,2, \Omega}$.

For the sake of simplicity of notation, let us denote by $X$ the product space $X=Y \times M$ and, by extension, $X_{h}=Y_{h} \times M_{h} \subset X$. We also define the $X$-norm as

$$
\|U\|_{X}=\sqrt{\|\mathbf{u}\|_{T}^{2}+\left\|p_{u}\right\|_{0,2, \Omega}^{2}} \quad \forall U=\left(\mathbf{u}, p_{u}\right) \in X .
$$

With this notation, we can rewrite the variational problem (2) as

$$
\left\{\begin{array}{l}
\text { Find } U_{h}(\mu) \in X_{h} \text { such that } \\
A\left(U_{h}(\mu), V_{h} ; \mu\right)=F\left(V_{h} ; \mu\right) \quad \forall V_{h} \in X_{h} .
\end{array}\right.
$$

In this formulation, the operator $A$ is given by

$$
A\left(U_{h}, V_{h} ; \mu\right)=\frac{1}{\mu} A_{0}\left(U_{h}, V_{h}\right)+A_{1}\left(U_{h}, V_{h}\right)+A_{2}\left(U_{h} ; V_{h}\right)+A_{3}\left(U_{h} ; V_{h}\right),
$$

where we denote $V_{h}=\left(\mathbf{v}_{h}, p_{v}\right)$, and

$$
\begin{aligned}
& A_{0}(U, V)=\int_{\Omega} \nabla \mathbf{u}: \nabla \mathbf{v} d \Omega, \quad A_{2}(U ; V)=\int_{\Omega}(\mathbf{u} \cdot \nabla \mathbf{u}) \mathbf{v} d \Omega \\
& A_{1}(U, V)=\int_{\Omega}\left[(\nabla \cdot \mathbf{u}) p_{v}-(\nabla \cdot \mathbf{v}) p_{u}\right] d \Omega+\int_{\Omega}\left(\mathbf{u}_{D} \cdot \nabla \mathbf{u}\right) \mathbf{v} d \Omega+\int_{\Omega}\left(\mathbf{u} \cdot \nabla \mathbf{u}_{D}\right) \mathbf{v} d \Omega \\
& A_{3}(U ; V)=\int_{\Omega} \nu_{T}\left(\mathbf{u}+\mathbf{u}_{D}\right) \nabla\left(\mathbf{u}+\mathbf{u}_{D}\right): \nabla \mathbf{v} d \Omega
\end{aligned}
$$

3. Reduced basis problem. In this section, we present the RB method for the Smagorinsky turbulence model. We will focus on the greedy algorithm. This is an adaptation of the RB method for Navier-Stokes equations (cf. [13, 22]). We assume 
that the Reynolds number ranges on a compact interval $\mathcal{D} \subset \mathbb{R}$. For the startup of the greedy algorithm, we choose an arbitrary parameter value $\mu^{1} \in \mathcal{D}_{\text {train }}$, and we compute the corresponding first snapshot $\left(\mathbf{u}_{h}\left(\mu^{1}\right), p_{h}\left(\mu^{1}\right)\right)$, solution of the finite element problem (5). Here $\mathcal{D}_{\text {train }} \subset \mathcal{D}$ is a discrete set where we select the possible values of $\mu$. We will denote by $N_{\max }$ the maximum number of basis functions.

In order to guarantee the inf-sup stability of the RB approximation $[4,31,32]$, let us consider the so-called inner pressure supremizer operator $T_{p}^{\mu}: M_{h} \rightarrow Y_{h}$, as

$$
\left(T_{p}^{\mu} q_{h}, \mathbf{v}_{h}\right)_{T}=b\left(q_{h}, \mathbf{v}_{h} ; \mu\right) \quad \forall \mathbf{v}_{h} \in Y_{h} .
$$

In this way, we define our first reduced velocity and pressure spaces as

$$
M_{1}=\operatorname{span}\left\{\xi_{1}^{p}:=p_{h}\left(\mu^{1}\right)\right\}, \quad Y_{1}=\operatorname{span}\left\{\zeta_{k}^{\mathbf{v}}:=\mathbf{u}_{h}\left(\mu^{1}\right), T_{p}^{\mu} \xi_{1}^{p}\right\} .
$$

To add a new element to the reduced space, choose the $(\mathrm{N}+1)$ th value of $\mu \in$ $\mathcal{D}_{\text {train }}$ as

$$
\mu^{N+1}=\arg \max _{\mu \in \mathcal{D}_{\text {train }}}\left\|U_{h}(\mu)-U_{N}(\mu)\right\|_{X}, \quad 1 \leq N \leq N_{\max }
$$

where $U_{N}(\mu)$ is the solution of the discrete model on the current RB space $X_{N}$ :

$$
\left\{\begin{array}{l}
\text { Given } \mu \in \mathcal{D}, \text { find } U_{N}(\mu) \in X_{N} \text { such that } \\
A\left(U_{N}(\mu), V_{N} ; \mu\right)=F\left(V_{N} ; \mu\right) \quad \forall V_{N} \in X_{N} .
\end{array}\right.
$$

Since the computation of $\left\|U_{h}(\mu)-U_{N}(\mu)\right\|_{X}$ may be very expensive due to the computation of the finite element solution $U_{h}(\mu)$ for all $\mu \in \mathcal{D}_{\text {train }}$, we consider an inexpensive a posteriori error estimator $\Delta_{N}$, constructed in section 5 , and we define

$$
\mu^{N+1}=\arg \max _{\mu \in \mathcal{D}_{\text {train }}} \Delta_{N}(\mu), \quad 1 \leq N \leq N_{\max } .
$$

Once we compute the optimum $\mu^{N+1}$, we add to the reduced space the new snapshots $\left(\mathbf{u}_{h}\left(\mu^{N+1}\right), p_{h}\left(\mu^{N+1}\right)\right)$, solution of the finite problem (5). We also have to add to the velocity space the supremizer corresponding to the pressure snapshot. Thus, the new reduced space, is $X_{N+1}=Y_{N+1} \times M_{N+1}$, where

$$
M_{N+1}=\operatorname{span}\left\{\xi_{k}^{p}:=p_{h}\left(\mu^{k}\right)\right\}_{k=1}^{N+1}, \quad Y_{N+1}=\operatorname{span}\left\{\zeta_{k}^{\mathbf{v}}:=\mathbf{u}_{h}\left(\mu^{k}\right), T_{p}^{\mu} \xi_{k}^{p}\right\}_{k=1}^{N+1} .
$$

Note that the construction of these spaces is hierarchical, i.e., $X_{1} \subset X_{2} \subset \cdots \subset$ $X_{N_{\max }} \subset X_{h}$. Finally, in order to avoid ill-conditioned matrices in the solution of (10), we orthonormalize the reduced velocity space $Y_{N}$ with respect the norm $\|\cdot\|_{T}$ and the reduced pressure space $M_{N}$ with respect the $L^{2}$-norm.

We summarize the greedy algorithm:

1. Set $\mu^{1}$, and compute $\mathbf{u}_{h}\left(\mu^{1}\right), p_{h}\left(\mu^{1}\right)$ and $T_{p}^{\mu} p_{h}\left(\mu^{1}\right)$, and the reduced spaces $Y_{1}, M_{1}$.

2. For $k \geq 2$, compute $\Delta_{k-1}(\mu)$ for all $\mu \in \mathcal{D}_{\text {train }}$ and set $\mu^{k}=$ $\arg \max _{\mu \in \mathcal{D}_{\text {train }}} \Delta_{k-1}(\mu)$.

3. Compute $\mathbf{u}_{h}\left(\mu^{k}\right), p_{h}\left(\mu^{k}\right)$ and $T_{p}^{\mu} p_{h}\left(\mu^{k}\right)$, and then the reduced spaces $Y_{k}, M_{k}$.

4. Stop if $\max _{\mu \in \mathcal{D}_{\text {train }}} \Delta_{k}(\mu)<\varepsilon_{R B}$. 
4. Well-posedness analysis. The well-posedness of the Smagorinsky problem is provided in [11] by the classical Brezzi theory (cf. [7]). The boundedness of the FE solution is provided by this analysis. However, in this section, we analyse the well-posedness of the Smagorinsky FE solution using the more general BRR theory (see, e.g., [8]). The finality of using the BRR theory instead the Brezzi theory is the construction of the error estimator provided by the BRR for the RB problem. Let us denote the directional derivative, at $U_{h} \in X_{h}$, in the direction $Z_{h}=\left(\mathbf{z}, p_{z}\right) \in X_{h}$, as $\partial_{1} A\left(U_{h}, \cdot ; \mu\right)\left(Z_{h}\right)$. If we derive each operator term in (6), we obtain

$$
\begin{aligned}
\partial_{1} A_{0}(U, V)(Z)= & A_{0}(Z, V), \quad \partial_{1} A_{1}(U, V)(Z)=A_{1}(Z, V) \\
\partial_{1} A_{2}(U ; V)(Z)= & \int_{\Omega}(\mathbf{u} \cdot \nabla \mathbf{z}) \mathbf{v} d \Omega+\int_{\Omega}(\mathbf{z} \cdot \nabla \mathbf{u}) \mathbf{v} d \Omega \\
\partial_{1} A_{3}(U ; V)(Z)= & \int_{\Omega} \nu_{T}\left(\mathbf{u}+\mathbf{u}_{D}\right) \nabla \mathbf{z}: \nabla \mathbf{v} d \Omega \\
& +\sum_{K \in \mathcal{T}} \int_{K}\left(C_{S} h_{K}\right)^{2} \frac{\nabla\left(\mathbf{u}+\mathbf{u}_{D}\right): \nabla \mathbf{z}}{\left|\nabla\left(\mathbf{u}+\mathbf{u}_{D}\right)\right|}\left(\nabla\left(\mathbf{u}+\mathbf{u}_{D}\right): \nabla \mathbf{v}\right) d \Omega
\end{aligned}
$$

For the well posedness of the problem, we have to guarantee the uniform coerciveness and the boundedness of $\partial_{1} A$ in the sense that for any solution $U_{h}(\mu)$ of $(5)$, there exist $\beta_{0}>0$ and $\gamma_{0} \in \mathbb{R}$ such that for all $\mu \in \mathcal{D}$,

$$
\begin{aligned}
& 0<\beta_{0}<\beta_{h}(\mu) \equiv \inf _{Z_{h} \in X_{h}} \sup _{V_{h} \in X_{h}} \frac{\partial_{1} A\left(U_{h}(\mu), V_{h} ; \mu\right)\left(Z_{h}\right)}{\left\|Z_{h}\right\|_{X}\left\|V_{h}\right\|_{X}}, \\
& \infty>\gamma_{0}>\gamma_{h}(\mu) \equiv \sup _{Z_{h} \in X_{h}} \sup _{V_{h} \in X_{h}} \frac{\partial_{1} A\left(U_{h}(\mu), V_{h} ; \mu\right)\left(Z_{h}\right)}{\left\|Z_{h}\right\|_{X}\left\|V_{h}\right\|_{X}} .
\end{aligned}
$$

Then, according to the BRR theory (cf. $[8,9])$, it will follow that in a neighborhood of $U_{h}(\mu)$ the solution of (5) is unique and bounded in $\|\cdot\|_{X}$ in terms of the data. We will prove this in section 5 , and as consequence we shall construct the a posteriori error bound estimator.

Since $H^{1}(\Omega)$ is embedded in $L^{4}(\Omega)$, let us denote by $C_{T}$ the Sobolev embedding constant such that $\|\mathbf{v}\|_{0,4, \Omega} \leq C_{T}\|\mathbf{v}\|_{T}$ for all $\mathbf{v} \in Y$. Also, let us denote by $C_{\bar{\mu}}$ the constant such that $\|\mathbf{v}\|_{T} \leq C_{\bar{\mu}}\|\nabla \mathbf{v}\|_{0,2, \Omega}$ for all $\mathbf{v} \in Y$. These constant will be used in the following propositions. By standard arguments, Proposition 4.1 follows.

Proposition 4.1. There exists $\gamma_{0} \in \mathbb{R}$ such that for all $\mu \in \mathcal{D}$

$$
\left|\partial_{1} A\left(U_{h}(\mu), V_{h} ; \mu\right)\left(Z_{h}\right)\right| \leq \gamma_{0}\left\|Z_{h}\right\|_{X}\left\|V_{h}\right\|_{X} \quad \forall Z_{h}, V_{h} \in X_{h} .
$$

Proving that the $\beta_{h}(\mu)$ inf-sup condition in (13) is satisfied, we ensure that we are in a smooth branch of solutions for the Smagorinsky problem.

Proposition 4.2. Let $C^{\star}=C_{T}^{2}\left(C_{\bar{\mu}}+1\right)$. Suppose that $\left\|\nabla \boldsymbol{u}_{D}\right\|_{0,2, \Omega}<\frac{1}{C^{\star}}$, and $\left\|\nabla \boldsymbol{u}_{h}\right\|_{0,2, \Omega} \leq \frac{1}{C^{\star}}-\left\|\nabla \boldsymbol{u}_{D}\right\|_{0,2, \Omega}$. Then, there exists $\tilde{\beta}_{h}>0$ such that

$$
\partial_{1} A\left(U_{h}, V_{h} ; \mu\right)\left(V_{h}\right) \geq \tilde{\beta}_{h}\left\|\boldsymbol{v}_{h}\right\|_{T}^{2} \quad \forall V_{h} \in X_{h} .
$$

Proof. We consider $Z_{h}=V_{h}$ in $\partial_{1} A\left(U_{h}, V_{h} ; \mu\right)\left(Z_{h}\right)$, having

$$
\begin{aligned}
\partial_{1} A\left(U_{h}, V_{h} ; \mu\right)\left(V_{h}\right)= & \frac{1}{\mu} \partial_{1} A_{0}\left(U_{h}, V_{h}\right)\left(V_{h}\right)+\partial_{1} A_{1}\left(U_{h}, V_{h}\right)\left(V_{h}\right) \\
& +\partial_{1} A_{2}\left(U_{h}, V_{h}\right)\left(V_{h}\right)+\partial_{1} A_{3}\left(U_{h} ; V_{h}\right)\left(V_{h}\right) .
\end{aligned}
$$

Copyright (c) by SIAM. Unauthorized reproduction of this article is prohibited. 
As

$$
\begin{aligned}
& \frac{1}{\mu} \partial_{1} A_{0}\left(U_{h}, V_{h}\right)\left(V_{h}\right)+\partial_{1} A_{3}\left(W_{h} ; V_{h}\right)\left(V_{h}\right) \\
& =\int_{\Omega}\left(\frac{1}{\mu}+\nu_{T}\left(\mathbf{w}_{h}\right)\right)\left|\nabla \mathbf{v}_{h}\right|^{2} d \Omega \\
& \quad+\sum_{K \in \mathcal{T}} \int_{K}\left(C_{S} h_{K}\right)^{2} \frac{\left|\nabla \mathbf{w}_{h}: \nabla \mathbf{w}_{h}\right|^{2}}{\left|\nabla \mathbf{w}_{h}\right|} d \Omega, \\
& \partial_{1} A_{1}\left(U_{h}, V_{h}\right)\left(V_{h}\right)+\partial_{1} A_{2}\left(U_{h} ; V_{h}\right)\left(V_{h}\right) \\
& =\int_{\Omega}\left(\mathbf{w}_{h} \cdot \nabla \mathbf{v}_{h}\right) \mathbf{v}_{h} d \Omega+\int_{\Omega}\left(\mathbf{v}_{h} \cdot \nabla \mathbf{w}_{h}\right) \mathbf{v}_{h} d \Omega \\
& \leq C_{T}^{2}\left(\left\|\mathbf{w}_{h}\right\|_{T}+\left\|\nabla \mathbf{w}_{h}\right\|_{0,2, \Omega}\right)\left\|\mathbf{v}_{h}\right\|_{T}^{2} \leq C_{T}^{2}\left(C_{\bar{\mu}}+1\right)\left\|\nabla \mathbf{w}_{h}\right\|_{0,2, \Omega}\left\|\mathbf{v}_{h}\right\|_{T}^{2} .
\end{aligned}
$$

Since $\sum_{K \in \mathcal{T}} \int_{K}\left(C_{S} h_{K}\right)^{2} \frac{\left|\nabla \mathbf{w}_{h}: \nabla \mathbf{v}_{h}\right|^{2}}{\left|\nabla \mathbf{w}_{h}\right|} d \Omega \geq 0$, we have, thanks to (16) and (17),

$$
\begin{aligned}
\partial_{1} A\left(U_{h}, V_{h} ; \mu\right)\left(V_{h}\right) \\
\quad \geq \int_{\Omega}\left(\frac{1}{\mu}+\nu_{T}\left(\mathbf{w}_{h}\right)\right)\left|\nabla \mathbf{v}_{h}\right|^{2} d \Omega \\
\quad-C_{T}^{2}\left(C_{\bar{\mu}}+1\right)\left\|\nabla \mathbf{w}_{h}\right\|_{0,2, \Omega}\left\|\mathbf{v}_{h}\right\|_{T}^{2} \geq\left(1-C_{T}^{2}\left(C_{\bar{\mu}}+1\right)\left\|\nabla \mathbf{w}_{h}\right\|_{0,2, \Omega}\right)\left\|\mathbf{v}_{h}\right\|_{T}^{2} \\
\quad \geq\left(1-C_{T}^{2}\left(C_{\bar{\mu}}+1\right)\left\|\nabla \mathbf{u}_{D}\right\|_{0,2, \Omega}-C_{T}^{2}\left(C_{\bar{\mu}}+1\right)\left\|\nabla \mathbf{u}_{h}\right\|_{0,2, \Omega}\right)\left\|\mathbf{v}_{h}\right\|_{T}^{2} .
\end{aligned}
$$

Thus, if $\left\|\nabla \mathbf{u}_{h}\right\|_{0,2, \Omega} \leq \frac{1}{C^{\star}}-\left\|\mathbf{u}_{D}\right\|_{0,2, \Omega}$ and $\left\|\mathbf{u}_{D}\right\|_{0,2, \Omega} \leq \frac{1}{C^{\star}}$, there exists $\beta_{h}>0$ such that

$$
\partial_{1} A\left(U_{h}, V_{h} ; \mu\right)\left(V_{h}\right) \geq \tilde{\beta_{h}}\left\|\mathbf{v}_{h}\right\|_{T}^{2} \quad \forall V_{h} \in X_{h} .
$$

Remark 4.3. Since the operator $b\left(\mathbf{v}_{h}, q_{h} ; \mu\right)$ satisfies the discrete inf-sup condition $\alpha\left\|q_{h}\right\|_{0,2, \Omega} \leq \sup _{\mathbf{v}_{h} \in Y_{h}} \frac{b\left(\mathbf{v}_{h}, p_{h} ; \mu\right)}{\|\mathbf{v}\|_{1,2, \Omega}}$, and thanks to Proposition 4.2, we can prove that the operator $\partial_{1} A$ satisfies the inf-sup condition in (13). See [10] for more details.

Observe that as $\left\|\mathbf{g}_{D}\right\|_{1 / 2, \Gamma_{D}} \leq\left\|\mathbf{w}_{h}\right\|_{1, \Omega} \leq C_{\Omega}\left\|\nabla \mathbf{w}_{h}\right\|_{0,2, \Omega}$, the condition needed in Proposition 4.2, $\left\|\nabla \mathbf{w}_{h}\right\|_{0,2, \Omega} \leq \frac{1}{C_{T}^{2}\left(C_{\bar{\mu}}+1\right)}$, will only be possible if $\left\|\mathbf{g}_{D}\right\|_{1 / 2, \Gamma_{D}} \leq$ $\frac{C_{\Omega}}{C_{T}^{2}\left(C_{\bar{\mu}}+1\right)}$; thus the Dirichlet boundary data should be sufficiently small.

5. A posteriori error estimator. In this section we construct the a posteriori error bound estimator for the greedy algorithm, which selects the snapshots for the reduced space $X_{N}$. In order to obtain this a posteriori error bound estimator, we will take into account the well-posedness analysis of the reduced problem (5) done in the previous section. We start by proving that the directional derivative of the operator $A(\cdot, \cdot ; \mu)$ is locally lipschitz.

Lemma 5.1. There exists a positive constant $\rho_{T}$ such that for all $U_{h}^{1}, U_{h}^{2}, Z_{h}, V_{h} \in$ $X_{h}$,

$$
\left|\partial_{1} A\left(U_{h}^{1}, V_{h} ; \mu\right)\left(Z_{h}\right)-\partial_{1} A\left(U_{h}^{2}, V_{h} ; \mu\right)\left(Z_{h}\right)\right| \leq \rho_{T}\left\|U_{h}^{1}-U_{h}^{2}\right\|_{X}\left\|Z_{h}\right\|_{X}\left\|V_{h}\right\|_{X} .
$$


Proof. We have that

$$
\begin{aligned}
\partial_{1} A & \left(U_{h}^{1}, V_{h} ; \mu\right)\left(Z_{h}\right)-\partial_{1} A\left(U_{h}^{2}, V_{h} ; \mu\right)\left(Z_{h}\right) \\
= & \int_{\Omega}\left(\left(\mathbf{u}_{h}^{1}-\mathbf{u}_{h}^{2}\right) \cdot \nabla \mathbf{z}_{h}\right) \mathbf{v}_{h} d \Omega \\
& +\int_{\Omega}\left(\mathbf{z}_{h} \cdot \nabla\left(\mathbf{u}_{h}^{1}-\mathbf{u}_{h}^{2}\right)\right) \mathbf{v}_{h} d \Omega+\int_{\Omega}\left(\nu_{T}\left(\mathbf{u}_{h}^{1}\right)-\nu_{T}\left(\mathbf{u}_{h}^{2}\right)\right) \nabla \mathbf{z}_{h}: \nabla \mathbf{v}_{h} d \Omega \\
& +\sum_{K \in \mathcal{T}_{h}} \int_{K}\left(C_{S} h_{K}\right)^{2} \frac{\nabla \mathbf{u}_{h}^{1}: \nabla \mathbf{z}_{h}}{\left|\nabla \mathbf{u}_{h}^{1}\right|}\left(\nabla \mathbf{u}_{h}^{1}: \nabla \mathbf{v}_{h}\right) d \Omega \\
& -\sum_{K \in \mathcal{T}_{h}} \int_{K}\left(C_{S} h_{K}\right)^{2} \frac{\nabla \mathbf{u}_{h}^{2}: \nabla \mathbf{z}_{h}}{\left|\nabla \mathbf{u}_{h}^{2}\right|}\left(\nabla \mathbf{u}_{h}^{2}: \nabla \mathbf{v}_{h}\right) d \Omega .
\end{aligned}
$$

So, thanks to the triangle inequality, it follows that

$$
\begin{aligned}
\mid \partial_{1} A & \left(U_{h}^{1}, V_{h} ; \mu\right)\left(Z_{h}\right)-\partial_{1} A\left(U_{h}^{2}, V_{h} ; \mu\right)\left(Z_{h}\right) \mid \\
\leq & \left|\int_{\Omega}\left(\left(\mathbf{u}_{h}^{1}-\mathbf{u}_{h}^{2}\right) \cdot \nabla \mathbf{z}_{h}\right) \mathbf{v}_{h} d \Omega\right| \\
+ & \left|\int_{\Omega}\left(\mathbf{z}_{h} \cdot \nabla\left(\mathbf{u}_{h}^{1}-\mathbf{u}_{h}^{2}\right)\right) \mathbf{v}_{h} d \Omega\right| \\
+ & \left|\sum_{K \in \mathcal{T}_{h}} \int_{K}\left(C_{S} h_{K}\right)^{2}\left(\left|\nabla \mathbf{u}_{h}^{1}\right|-\left|\nabla \mathbf{u}_{h}^{2}\right|\right) \nabla \mathbf{z}_{h}: \nabla \mathbf{v}_{h} d \Omega\right| \\
+ & \mid \sum_{K \in \mathcal{T}_{h}} \int_{K}\left(C_{S} h_{K}\right)^{2} \frac{\nabla \mathbf{u}_{h}^{1}: \nabla \mathbf{z}_{h}}{\left|\nabla \mathbf{u}_{h}^{1}\right|}\left(\nabla \mathbf{u}_{h}^{1}: \nabla \mathbf{v}_{h}\right) d \Omega \\
& \quad-\sum_{K \in \mathcal{T}_{h}} \int_{K}\left(C_{S} h_{K}\right)^{2} \frac{\nabla \mathbf{u}_{h}^{2}: \nabla \mathbf{z}_{h}}{\left|\nabla \mathbf{u}_{h}^{2}\right|}\left(\nabla \mathbf{u}_{h}^{2}: \nabla \mathbf{v}_{h}\right) d \Omega \mid .
\end{aligned}
$$

We bound each term separately in (20). For the first two terms, we use the relation between $\|\cdot\|_{0,4, \Omega}$ and $\|\cdot\|_{T}$ used in Proposition 4.1 and the fact that the T-norm is equivalent to the $H^{1}$-seminorm.

$$
\begin{aligned}
& \left|\int_{\Omega}\left(\left(\mathbf{u}_{h}^{1}-\mathbf{u}_{h}^{2}\right) \cdot \nabla \mathbf{z}_{h}\right) \mathbf{v}_{h} d \Omega\right| \\
& \quad \leq \int_{\Omega}\left|\mathbf{u}_{h}^{1}-\mathbf{u}_{h}^{2}\right| \mid \nabla \mathbf{z}_{h}\left\|\mathbf{v}_{h}\right\| d \Omega \\
& \quad \leq\left\|\mathbf{u}_{h}^{1}-\mathbf{u}_{h}^{2}\right\|_{0,4, \Omega}\left\|\nabla \mathbf{z}_{h}\right\|_{0,2, \Omega}\left\|\mathbf{v}_{h}\right\|_{0,4, \Omega} \leq C_{T}\left\|\mathbf{u}_{h}^{1}-\mathbf{u}_{h}^{2}\right\|_{T}\left\|\mathbf{z}_{h}\right\|_{T}\left\|\mathbf{v}_{h}\right\|_{T} \\
& \quad \leq C_{T}\left\|U_{h}^{1}-U_{h}^{2}\right\|_{X}\left\|Z_{h}\right\|_{X}\left\|V_{h}\right\|_{X} \\
& \left|\int_{\Omega}\left(\mathbf{z}_{h} \cdot \nabla\left(\mathbf{u}_{h}^{1}-\mathbf{u}_{h}^{2}\right)\right) \mathbf{v}_{h} d \Omega\right| \\
& \quad \leq \int_{\Omega}\left|\mathbf{z}_{h}\left\|\nabla\left(\mathbf{u}_{h}^{1}-\mathbf{u}_{h}^{2}\right)\right\| \mathbf{v}_{h}\right| d \Omega \\
& \quad \leq\left\|\mathbf{z}_{h}\right\|_{0,4, \Omega}\left\|\nabla\left(\mathbf{u}_{h}^{1}-\mathbf{u}_{h}^{2}\right)\right\|_{0,2, \Omega}\left\|\mathbf{v}_{h}\right\|_{0,4, \Omega} \leq C_{T}\left\|\mathbf{z}_{h}\right\|_{T}\left\|\mathbf{u}^{1}-\mathbf{u}_{h}^{2}\right\|_{T}\left\|\mathbf{v}_{h}\right\|_{T} \\
& \quad \leq C_{T}\left\|Z_{h}\right\|_{X}\left\|U_{h}^{1}-U_{h}^{2}\right\|_{X}\left\|V_{h}\right\|_{X} .
\end{aligned}
$$

Copyright (c) by SIAM. Unauthorized reproduction of this article is prohibited. 
To bound the third term in (20), we use the local inverse inequalities (cf. [6]),

$$
\begin{aligned}
& \left|\sum_{K \in \mathcal{T}_{h}} \int_{K}\left(C_{S} h_{K}\right)^{2}\left(\left|\nabla \mathbf{u}_{h}^{1}\right|-\left|\nabla \mathbf{u}_{h}^{2}\right|\right) \nabla \mathbf{z}_{h}: \nabla \mathbf{v}_{h} d \Omega\right| \\
& \quad \leq \sum_{K \in \mathcal{T}_{h}} \int_{K}\left(C_{S} h_{K}\right)^{2} \| \nabla \mathbf{u}_{h}^{1}|-| \nabla \mathbf{u}_{h}^{2}||\left|\nabla \mathbf{z}_{h}\right|\left|\nabla \mathbf{v}_{h}\right| d \Omega \\
& \quad \leq\left(C_{S} h\right)^{2} \int_{\Omega}\left|\nabla\left(\mathbf{u}_{h}^{1}-\mathbf{u}_{h}^{2}\right) \| \nabla \mathbf{z}_{h}\right|\left|\nabla \mathbf{v}_{h}\right| d \Omega \\
& \quad \leq\left(C_{S} h\right)^{2}\left\|\nabla\left(\mathbf{u}_{h}^{1}-\mathbf{u}_{h}^{2}\right)\right\|_{0,3, \Omega}\left\|\nabla \mathbf{z}_{h}\right\|_{0,3, \Omega}\left\|\nabla \mathbf{v}_{h}\right\|_{0,3, \Omega} \\
& \quad \leq C_{S}^{2} h^{2-d / 2} C\left\|\nabla\left(\mathbf{u}_{h}^{1}-\mathbf{u}_{h}^{2}\right)\right\|_{0,2, \Omega}\left\|\nabla \mathbf{z}_{h}\right\|_{0,2, \Omega}\left\|\nabla \mathbf{v}_{h}\right\|_{0,2, \Omega} \\
& \quad \leq C_{S}^{2} h^{2-d / 2} C\left\|\mathbf{u}_{h}^{1}-\mathbf{u}_{h}^{2}\right\|_{X}\left\|\mathbf{z}_{h}\right\|_{X}\left\|\mathbf{v}_{h}\right\|_{X} .
\end{aligned}
$$

The last term in (20) is bounded as follows:

$$
\begin{aligned}
& \mid \sum_{K \in \mathcal{T}_{h}} \int_{K}\left(C_{S} h_{K}\right)^{2} \frac{\nabla \mathbf{u}_{h}^{1}: \nabla \mathbf{z}_{h}}{\left|\nabla \mathbf{u}_{h}^{1}\right|}\left(\nabla \mathbf{u}_{h}^{1}: \nabla \mathbf{v}_{h}\right) d \Omega \\
& -\sum_{K \in \mathcal{T}_{h}} \int_{K}\left(C_{S} h_{K}\right)^{2} \frac{\nabla \mathbf{u}_{h}^{2}: \nabla \mathbf{z}_{h}}{\left|\nabla \mathbf{u}_{h}^{2}\right|}\left(\nabla \mathbf{u}_{h}^{2}: \nabla \mathbf{v}_{h}\right) d \Omega \mid \\
& =\mid \sum_{K \in \mathcal{T}_{h}} \int_{K}\left(C_{S} h_{K}\right)^{2}\left[\frac{\nabla \mathbf{u}_{h}^{1}: \nabla \mathbf{z}_{h}}{\left|\nabla \mathbf{u}_{h}^{1}\right|}\left(\nabla\left(\mathbf{u}_{h}^{1}-\mathbf{u}_{h}^{2}\right): \nabla \mathbf{v}_{h}\right)\right. \\
& \left.+\frac{\nabla\left(\mathbf{u}_{h}^{1}-\mathbf{u}_{h}^{2}\right): \nabla \mathbf{z}_{h}}{\left|\nabla \mathbf{u}_{h}^{2}\right|}\left(\nabla \mathbf{u}_{h}^{2}: \nabla \mathbf{v}_{h}\right)\right] d \Omega \\
& +\sum_{K \in \mathcal{T}_{h}} \int_{K}\left(C_{S} h_{K}\right)^{2} \frac{\left(\left|\nabla \mathbf{u}_{h}^{2}\right|-\left|\nabla \mathbf{u}_{h}^{1}\right|\right) \nabla \mathbf{u}_{h}^{1}: \nabla \mathbf{z}_{h}}{\left|\nabla \mathbf{u}_{h}^{1}\right|\left|\nabla \mathbf{u}_{h}^{2}\right|}\left(\nabla \mathbf{u}_{h}^{2}: \nabla \mathbf{v}_{h}\right) d \Omega \mid \\
& \leq \sum_{K \in \mathcal{T}_{h}} \int_{K}\left(C_{S} h_{K}\right)^{2}\left|\nabla \mathbf{z}_{h}\right|\left|\nabla\left(\mathbf{u}_{h}^{1}-\mathbf{u}_{h}^{2}\right)\right|\left|\nabla \mathbf{v}_{h}\right| d \Omega \\
& +\sum_{K \in \mathcal{T}_{h}} \int_{K}\left(C_{S} h_{K}\right)^{2}\left|\nabla\left(\mathbf{u}_{h}^{1}-\mathbf{u}_{h}^{2}\right)\right|\left|\nabla \mathbf{z}_{h}\right|\left|\nabla \mathbf{v}_{h}\right| d \Omega \\
& +\sum_{K \in \mathcal{T}_{h}} \int_{K}\left(C_{S} h_{K}\right)^{2}|| \nabla \mathbf{u}_{h}^{1}|-| \nabla \mathbf{u}_{h}^{2}||\left|\nabla \mathbf{z}_{h}\right|\left|\nabla \mathbf{v}_{h}\right| d \Omega \\
& \leq 3\left(C_{S} h\right)^{2}\left\|\nabla\left(\mathbf{u}_{h}^{1}-\mathbf{u}_{h}^{2}\right)\right\|_{0,3, \Omega}\left\|\nabla \mathbf{z}_{h}\right\|_{0,3, \Omega}\left\|\nabla \mathbf{v}_{h}\right\|_{0,3, \Omega} \\
& \leq 3 C_{S}^{2} h^{2-d / 2} C\left\|\nabla\left(\mathbf{u}_{h}^{1}-\mathbf{u}_{h}^{2}\right)\right\|_{0,2, \Omega}\left\|\nabla \mathbf{z}_{h}\right\|_{0,2, \Omega}\left\|\nabla \mathbf{v}_{h}\right\|_{0,2, \Omega} \\
& \leq 3 C_{S}^{2} h^{2-d / 2} C\left\|U_{h}^{1}-U_{h}^{2}\right\|_{X}\left\|Z_{h}\right\|_{X}\left\|V_{h}\right\|_{X} .
\end{aligned}
$$

Thus, we have just proved that $\left|\partial_{1} A\left(U_{h}^{1}, V_{h} ; \mu\right)\left(Z_{h}\right)-\partial_{1} A\left(U_{h}^{2}, V_{h} ; \mu\right)\left(Z_{h}\right)\right| \leq$ $\rho_{T}\left\|U_{h}^{1}-U_{h}^{2}\right\|_{X}\left\|Z_{h}\right\|_{X}\left\|V_{h}\right\|_{X}$, where, $\rho_{T}=2 C_{T}+4 C_{S} h^{2-d / 2} C$.

We introduce the following supremizer operator $T_{N}: X_{h} \rightarrow X_{h}$, defined as

$$
\left(T_{N} Z_{h}, V_{h}\right)_{X}=\partial_{1} A\left(U_{N}(\mu), V_{h} ; \mu\right)\left(Z_{h}\right) \quad \forall V_{h}, Z_{h} \in X_{h},
$$

such that

$$
T_{N} Z_{h}=\arg \sup _{V_{h} \in X_{h}} \frac{\partial_{1} A\left(U_{N}(\mu), V_{h} ; \mu\right)\left(Z_{h}\right)}{\left\|V_{h}\right\|_{X}} .
$$

Copyright (๑) by SIAM. Unauthorized reproduction of this article is prohibited. 
Taking this definition into account, in order to guarantee the well-posedness of the RB problem (10), in the same way as in the finite element problem (5), we define the inf-sup and continuity constants:

$$
\begin{aligned}
& 0<\beta_{N}(\mu) \equiv \inf _{Z_{h} \in X_{h}} \sup _{V_{h} \in X_{h}} \frac{\partial_{1} A\left(U_{N}(\mu), V_{h} ; \mu\right)\left(Z_{h}\right)}{\left\|Z_{h}\right\|_{X}\left\|V_{h}\right\|_{X}}=\inf _{Z_{h} \in X_{h}} \frac{\left\|T_{N} Z_{h}\right\|_{X}}{\left\|Z_{h}\right\|_{X}}, \\
& \infty>\gamma_{N}(\mu) \equiv \sup _{Z_{h} \in X_{h}} \sup _{V_{h} \in X_{h}} \frac{\partial_{1} A\left(U_{N}(\mu), V_{h}: \mu\right)\left(V_{h}\right)}{\left\|Z_{h}\right\|_{X}\left\|V_{h}\right\|_{X}}=\sup _{Z_{h} \in X_{h}} \frac{\left\|T_{N} Z_{h}\right\|_{X}}{\left\|Z_{h}\right\|_{X}} .
\end{aligned}
$$

THEOREM 5.2. Let $\mu \in \mathcal{D}$, and assume that $\beta_{N}(\mu)>0$. If problem (5) admits a solution $U_{h}(\mu)$ such that

$$
\left\|U_{h}(\mu)-U_{N}(\mu)\right\|_{X} \leq \frac{\beta_{N}(\mu)}{\rho_{T}}
$$

then this solution is unique in the ball $B_{X}\left(U_{N}(\mu), \frac{\beta_{N}(\mu)}{\rho_{T}}\right)$.

Proof. The proof of this theorem is an extension of the proof of [13, Lemma 3.1]. We define the following operators:

- $\mathcal{R}(\cdot ; \mu): X_{h} \rightarrow X_{h}^{\prime}$, as

$$
\left\langle\mathcal{R}\left(Z_{h} ; \mu\right), V_{h}\right\rangle=A\left(Z_{h}, V_{h} ; \mu\right)-F\left(V_{h} ; \mu\right) \quad \forall Z_{h}, V_{h} \in X_{h} .
$$

- $\mathcal{D} \mathcal{A}\left(U_{h}(\mu) ; \mu\right): X_{h} \rightarrow X_{h}^{\prime}$, defined, for $U_{h}(\mu) \in X_{h}$, as

$$
\left\langle\mathcal{D} \mathcal{A}\left(U_{h}(\mu) ; \mu\right) Z_{h}, V_{h}\right\rangle=\partial_{1} A\left(U_{h}(\mu), V_{h} ; \mu\right)\left(Z_{h}\right) \quad \forall Z_{h}, V_{h} \in X_{h} .
$$

- $H: X_{h} \rightarrow X_{h}$, defined as

$$
H\left(Z_{h} ; \mu\right)=Z_{h}-\mathcal{D} \mathcal{A}\left(U_{N}(\mu) ; \mu\right)^{-1} \mathcal{R}\left(Z_{h} ; \mu\right) \quad \forall Z_{h} \in X_{h} .
$$

Note that $\mathcal{D} \mathcal{A}\left(U_{N}(\mu) ; \mu\right)$ is invertible thanks to the assumption $\beta_{N}(\mu)>0$. Also note that $\mathcal{D} \mathcal{A}\left(U_{N}(\mu) ; \mu\right)=T_{N}$ in $X_{h}^{\prime}$. We express

(28)

$$
H\left(Z_{h}^{1} ; \mu\right)-H\left(Z_{h}^{2} ; \mu\right)=\left(Z_{h}^{1}-Z_{h}^{2}\right)-\mathcal{D} \mathcal{A}\left(U_{N}(\mu) ; \mu\right)^{-1}\left(\mathcal{R}\left(Z_{h}^{1} ; \mu\right)-\mathcal{R}\left(Z_{h}^{2} ; \mu\right)\right) .
$$

It holds that

$$
\mathcal{R}\left(Z_{h}^{1} ; \mu\right)-\mathcal{R}\left(Z_{h}^{2} ; \mu\right)=\mathcal{D} \mathcal{A}(\xi ; \mu)\left(Z_{h}^{1}-Z_{h}^{2}\right),
$$

where $\xi=\lambda Z_{h}^{1}-(1-\lambda) Z_{h}^{2}$ for some $\lambda \in(0,1)$. To prove this, we define the operator $T:[0,1] \rightarrow \mathbb{R}$ by $T(t)=\left\langle\mathcal{R}\left(t Z_{h}^{1}+(1-t) Z_{h}^{2} ; \mu\right), V_{h}\right\rangle$ for all $V_{h} \in X$. Then, $T(0)=$ $\left\langle\mathcal{R}\left(Z_{h}^{2} ; \mu\right), V_{h}\right\rangle$ and $T(1)=\left\langle\mathcal{R}\left(Z_{h}^{1} ; \mu\right), V_{h}\right\rangle$. The operator $T$ is differentiable in $(0,1)$ and continuous in $[0,1]$, and

$$
T^{\prime}(t)=\left\langle\mathcal{D} \mathcal{A}\left(t Z^{2}+(1-t) Z^{1} ; \mu\right)\left(Z_{h}^{1}-Z_{h}^{2}\right), V_{h}\right\rangle .
$$

Thus, (29) follows from the mean value theorem in $\mathbb{R}$. Now, multiplying (28) by $\mathcal{D} \mathcal{A}\left(U_{N}(\mu) ; \mu\right)$ and applying this last property, we can write

$$
\mathcal{D} \mathcal{A}\left(U_{N}(\mu) ; \mu\right)\left(H\left(Z_{h}^{1} ; \mu\right)-H\left(Z_{h}^{2} ; \mu\right)\right)=\left[\mathcal{D} \mathcal{A}\left(U_{N}(\mu) ; \mu\right)-\mathcal{D} \mathcal{A}(\xi ; \mu)\right]\left(Z_{h}^{1}-Z_{h}^{2}\right) .
$$

Then, thanks to (19) and this last equality, it follows that $\left\langle\mathcal{D} \mathcal{A}\left(U_{N}(\mu) ; \mu\right)\left(H\left(Z_{h}^{1} ; \mu\right)-H\left(Z_{h}^{2} ; \mu\right)\right), V_{h}\right\rangle \leq \rho_{T}\left\|U_{N}(\mu)-\xi\right\|_{X}\left\|Z_{h}^{1}-Z_{h}^{2}\right\|_{X}\left\|V_{h}\right\|_{X}$. 
Now, applying the definitions of $\beta_{N}(\mu), T_{N}, \mathcal{D} \mathcal{A}\left(U_{N}(\mu) ; \mu\right)$ and this last property, we can obtain

$$
\begin{aligned}
& \beta_{N}(\mu)\left\|H\left(Z_{h}^{1} ; \mu\right)-H\left(Z_{h}^{2} ; \mu\right)\right\|_{X}\left\|T_{N}\left(H\left(Z_{h}^{1} ; \mu\right)-H\left(Z_{h}^{2} ; \mu\right)\right)\right\|_{X} \\
& \quad \leq\left\|T_{N}\left(H\left(Z_{h}^{1} ; \mu\right)-H\left(Z_{h}^{2} ; \mu\right)\right)\right\|_{X}^{2} \\
& \quad=\left(T_{N}\left(H\left(Z_{h}^{1} ; \mu\right)-H\left(Z_{h}^{2} ; \mu\right) ; \mu\right), T_{N}\left(H\left(Z_{h}^{1} ; \mu\right)-H\left(Z_{h}^{2} ; \mu\right) ; \mu\right)\right)_{X} \\
& \quad=\left\langle\mathcal{D} \mathcal{A}\left(U_{N}(\mu) ; \mu\right)\left(H\left(Z_{h}^{1} ; \mu\right)-H\left(Z_{h}^{2} ; \mu\right)\right), T_{N}\left(H\left(Z_{h}^{1} ; \mu\right)-H\left(Z_{h}^{2} ; \mu\right) ; \mu\right)\right\rangle \\
& \quad \leq \rho_{T}\left\|U_{N}(\mu)-\xi\right\|_{X}\left\|Z_{h}^{1}-Z_{h}^{2}\right\|_{X}\left\|T_{N}\left(H\left(Z_{h}^{1} ; \mu\right)-H\left(Z_{h}^{2} ; \mu\right) ; \mu\right)\right\|_{X} .
\end{aligned}
$$

We have proved that $\left\|H\left(Z_{h}^{1} ; \mu\right)-H\left(Z_{h}^{2} ; \mu\right)\right\|_{X} \leq \frac{\rho_{T}}{\beta_{N}(\mu)}\left\|U_{N}(\mu)-\xi\right\|_{X}\left\|Z_{h}^{1}-Z_{h}^{2}\right\|_{X}$. If $Z^{1}$ and $Z^{2}$ are in $B_{X}\left(U_{N}(\mu), \alpha\right)$, then $\left\|U_{N}(\mu)-\xi\right\|_{X} \leq \alpha$ and

$$
\left\|H\left(Z_{h}^{1} ; \mu\right)-H\left(Z_{h}^{2} ; \mu\right)\right\|_{X} \leq \frac{\rho_{T}}{\beta_{N}(\mu)} \alpha\left\|Z_{h}^{1}-Z_{h}^{2}\right\|_{X} .
$$

Then, $H(\cdot ; \mu)$ is a contraction if $\alpha<\frac{\beta_{N}(\mu)}{\rho_{T}}$. So it follows that there can exist at most one fixed point of $H(\cdot ; \mu)$ inside $B_{X}\left(U_{N}(\mu), \frac{\beta_{N}(\mu)}{\rho_{T}}\right)$, and hence, at most one solution $U_{h}(\mu)$ to $(5)$ in this ball.

At this point, let us define the a posteriori error bound estimator by

$$
\Delta_{N}(\mu)=\frac{\beta_{N}(\mu)}{2 \rho_{T}}\left[1-\sqrt{1-\tau_{N}(\mu)}\right]
$$

where $\tau_{N}(\mu)$ is given by

$$
\tau_{N}(\mu)=\frac{4 \epsilon_{N}(\mu) \rho_{T}}{\beta_{N}^{2}(\mu)}
$$

with

$$
\epsilon_{N}(\mu)=\left\|\mathcal{R}\left(U_{N}(\mu) ; \mu\right)\right\|_{X^{\prime}} .
$$

The suitability of this a posteriori error bound estimator is stated by the following.

Theorem 5.3. Assume that $\beta_{N}(\mu)>0$ and $\tau_{N}(\mu) \leq 1$ for all $\mu \in \mathcal{D}$. Then there exists a unique solution $U_{h}(\mu)$ of $(5)$ such that the error with respect $U_{N}(\mu)$, solution of (10), is bounded by the a posteriori error bound estimator, i.e.,

$$
\left\|U_{h}(\mu)-U_{N}(\mu)\right\|_{X} \leq \Delta_{N}(\mu)
$$

with effectivity

$$
\Delta_{N}(\mu) \leq\left[\frac{2 \gamma_{N}(\mu)}{\beta_{N}(\mu)}+\tau_{N}(\mu)\right]\left\|U_{h}(\mu)-U_{N}(\mu)\right\|_{X} .
$$

Proof. To prove (33), let $\alpha>0$ and $Z_{h} \in X_{h}$ such that $\left\|U_{N}(\mu)-Z_{h}\right\|_{X} \leq \alpha$. We use the notation introduced in the proof of Theorem 5.2. We consider

$$
\begin{aligned}
H\left(Z_{h} ; \mu\right)-U_{N}(\mu)= & Z_{h}-U_{N}(\mu)-\mathcal{D} \mathcal{A}\left(U_{N}(\mu) ; \mu\right)^{-1} \mathcal{R}\left(Z_{h} ; \mu\right) \\
= & Z_{h}-U_{N}(\mu)-\mathcal{D} \mathcal{A}\left(U_{N}(\mu) ; \mu\right)^{-1}\left[\mathcal{R}\left(Z_{h} ; \mu\right)-\mathcal{R}\left(U_{N}(\mu) ; \mu\right)\right] \\
& -\mathcal{D} \mathcal{A}\left(U_{N}(\mu) ; \mu\right)^{-1} \mathcal{R}\left(U_{N}(\mu) ; \mu\right)
\end{aligned}
$$

Copyright (c) by SIAM. Unauthorized reproduction of this article is prohibited. 
Multiplying by $\mathcal{D} \mathcal{A}\left(U_{N}(\mu) ; \mu\right)$, we obtain

$$
\begin{aligned}
\langle\mathcal{D} \mathcal{A} & \left.\left(U_{N}(\mu) ; \mu\right)\left(H\left(Z_{h} ; \mu\right)-U_{N}(\mu)\right), V_{h}\right\rangle \\
= & \left\langle\mathcal{D} \mathcal{A}\left(U_{N}(\mu) ; \mu\right)\left(Z_{h}-U_{N}(\mu)\right), V_{h}\right\rangle \\
& -\left\langle\mathcal{R}\left(Z_{h} ; \mu\right)-\mathcal{R}\left(U_{N}(\mu) ; \mu\right), V_{h}\right\rangle-\left\langle\mathcal{R}\left(U_{N}(\mu) ; \mu\right), V_{h}\right\rangle \quad \forall V_{h} \in X_{h} .
\end{aligned}
$$

As in the proof of Theorem 5.2, it holds that

$$
\mathcal{R}\left(Z_{h} ; \mu\right)-\mathcal{R}\left(U_{N}(\mu) ; \mu\right)=\mathcal{D} \mathcal{A}(\xi(\mu) ; \mu)\left(Z_{h}-U_{N}(\mu)\right),
$$

where $\xi(\mu)=t^{*} Z_{h}+\left(1-t^{*}\right) U_{N}(\mu), t^{*} \in(0,1)$.

Due to this and Lemma 5.1, we obtain

$$
\begin{aligned}
\langle\mathcal{D} & \left.\mathcal{A}\left(U_{N}(\mu) ; \mu\right)\left(H\left(Z_{h} ; \mu\right)-U_{N}(\mu)\right), V_{h}\right\rangle \\
= & \left\langle\mathcal{D} \mathcal{A}\left(U_{N}(\mu) ; \mu\right)\left(Z_{h}-U_{N}(\mu)\right), V_{h}\right\rangle \\
& -\left\langle\mathcal{D} \mathcal{A}(\xi(\mu) ; \mu)\left(Z_{h}-U_{N}(\mu)\right), V_{h}\right\rangle-\left\langle\mathcal{R}\left(U_{N}(\mu) ; \mu\right), V_{h}\right\rangle \\
= & \left\langle\left(\mathcal{D} \mathcal{A}\left(U_{N}(\mu) ; \mu\right)-\mathcal{D} \mathcal{A}(\xi(\mu) ; \mu)\right)\left(Z_{h}-U_{N}(\mu)\right), V_{h}\right\rangle-\left\langle\mathcal{R}\left(U_{N}(\mu) ; \mu\right), V_{h}\right\rangle \\
\leq & \rho_{T}\left\|U_{N}(\mu)-\xi(\mu)\right\|_{X}\left\|Z_{h}-U_{N}(\mu)\right\|_{X}\left\|V_{h}\right\|_{X}+\epsilon_{N}(\mu)\left\|V_{h}\right\|_{X} \\
\leq & \left(\rho_{T}\left\|Z_{h}-U_{N}(\mu)\right\|_{X}^{2}+\epsilon_{N}(\mu)\right)\left\|V_{h}\right\|_{X} .
\end{aligned}
$$

Then, using the same arguments as in Theorem 5.2,

$$
\begin{aligned}
\beta_{N} & (\mu)\left\|H\left(Z_{h} ; \mu\right)-U_{N}(\mu)\right\|_{X}\left\|T_{N}^{\mu}\left(H\left(Z_{h} ; \mu\right)-U_{N}(\mu)\right)\right\|_{X} \\
& \leq\left\|T_{N}^{\mu}\left(H\left(Z_{h} ; \mu\right)-U_{N}(\mu)\right)\right\|_{X}^{2} \\
& =\left(T_{N}^{\mu}\left(H\left(Z_{h} ; \mu\right)-U_{N}(\mu)\right), T_{N}^{\mu}\left(H\left(Z_{h} ; \mu\right)-U_{N}(\mu)\right)\right)_{X} \\
& =\left\langle\mathcal{D} \mathcal{A}\left(U_{N}(\mu) ; \mu\right)\left(H\left(Z_{h} ; \mu\right)-U_{N}(\mu)\right), T_{N}^{\mu}\left(H\left(Z_{h} ; \mu\right)-U_{N}(\mu)\right)\right\rangle \\
& \leq\left(\rho_{T}\left\|Z_{h}-U_{N}(\mu)\right\|_{X}^{2}+\epsilon_{N}(\mu)\right)\left\|T_{N}^{\mu}\left(H\left(Z_{h} ; \mu\right)-U_{N}(\mu)\right)\right\|_{X} .
\end{aligned}
$$

Then, as $Z_{h} \in B_{X}\left(U_{N}(\mu), \alpha\right)$, we have

$$
\left\|H\left(Z_{h} ; \mu\right)-U_{N}(\mu)\right\|_{X}<\frac{\rho_{T}}{\beta_{N}(\mu)} \alpha^{2}+\frac{\epsilon_{N}(\mu)}{\beta_{N}(\mu)} .
$$

In order to ensure that $H$ maps $B_{X}\left(U_{N}(\mu), \alpha\right)$ into a part of itself, we are seeking the values of $\alpha$ such that $\frac{\rho_{T}}{\beta_{N}(\mu)} \alpha^{2}+\frac{\epsilon_{N}(\mu)}{\beta_{N}(\mu)} \leq \alpha$. This holds if $\alpha$ is between the two roots of the second order equation $\rho_{T} \alpha^{2}-\beta_{N}(\mu) \alpha+\epsilon_{N}(\mu)=0$, which are

$$
\alpha_{ \pm}=\frac{\beta_{N}(\mu) \pm \sqrt{\beta_{N}(\mu)^{2}-4 \rho_{T} \epsilon_{N}(\mu)}}{2 \rho_{T}}=\frac{\beta_{N}(\mu)}{2 \rho_{T}}\left[1 \pm \sqrt{1-\frac{4 \rho_{T} \epsilon_{N}(\mu)}{\beta_{N}(\mu)^{2}}}\right] .
$$

Observe that as $\tau_{N}(\mu) \leq 1, \alpha_{-} \leq \alpha_{+} \leq \frac{\beta_{N}(\mu)}{\rho_{T}}$. Consequently, if $\alpha_{-} \leq \alpha \leq \alpha_{+}$, there exists a unique solution $U_{h}(\mu)$ to $(5)$ in the ball $B_{X}\left(U_{N}(\mu), \alpha\right)$.

To obtain (33) observe that from (36), the lowest value (i.e., the best error bound) corresponds to $\alpha=\alpha_{-}=\Delta_{N}(\mu)$. To prove (34), let us define the error $E_{h}(\mu)=$ $U_{h}(\mu)-U_{N}(\mu)$, and the residual $R(\mu)$, such that

$$
\begin{aligned}
\left(R(\mu), V_{h}\right)_{X} & =-\left\langle\mathcal{R}\left(U_{N}(\mu) ; \mu\right), V_{h}\right\rangle=F\left(V_{h} ; \mu\right)-A\left(U_{N}(\mu), V_{h} ; \mu\right) \\
& =A\left(U_{h}(\mu), V_{;} \mu\right)-A\left(U_{N}(\mu), V_{h} ; \mu\right) .
\end{aligned}
$$

Copyright (c) by SIAM. Unauthorized reproduction of this article is prohibited. 
Note that, from (32), $\|R(\mu)\|_{X}=\epsilon_{N}(\mu)$. We observe that the following relation holds for some $t^{*} \in(0,1)$ :

$$
A\left(U_{h}(\mu), V_{h} ; \mu\right)-A\left(U_{N}(\mu), V_{h} ; \mu\right)=\partial_{1} A\left(t^{*} U_{h}(\mu)+\left(1-t^{*}\right) U_{N}(\mu), V_{h} ; \mu\right)\left(E_{h}(\mu)\right) .
$$

Thus, we have that

$$
\begin{aligned}
\|R(\mu)\|_{X}^{2}= & {\left[\partial_{1} A\left(t^{*} U_{h}(\mu)+\left(1-t^{*}\right) U_{N}(\mu), R(\mu) ; \mu\right)-\partial_{1} A\left(U_{N}(\mu), R(\mu) ; \mu\right)\right]\left(E_{h}(\mu)\right) } \\
& +\partial_{1} A\left(U_{N}(\mu), R(\mu) ; \mu\right)\left(E_{h}(\mu)\right) .
\end{aligned}
$$

Thus, thanks to Lemma 5.1, and taking into account the definition of $\gamma_{N}(\mu)$ by (24), we obtain

$$
\|R(\mu)\|_{X}^{2} \leq \rho_{T}\left\|t^{*}\left(U_{h}(\mu)-U_{N}(\mu)\right)\right\|_{X}\left\|E_{h}(\mu)\right\|_{X}\|R(\mu)\|_{X}+\gamma_{N}(\mu)\left\|E_{h}(\mu)\right\|_{X}\|R(\mu)\|_{X} .
$$

Then $\epsilon_{N}(\mu)=\rho_{T}\|E(\mu)\|_{X}^{2}+\|R(\mu)\|_{X} \leq \gamma_{N}(\mu)\|E(\mu)\|_{X}$. Since $0 \leq \tau_{N}(\mu) \leq 1$ we have that

$$
\frac{2 \rho_{T}}{\beta_{N}(\mu)} \Delta_{N}(\mu) \leq \tau_{N}(\mu)
$$

and then $\Delta_{N}(\mu) \leq 2 \epsilon_{N}(\mu) / \beta_{N}(\mu)$. It follows that

$$
\Delta_{N}(\mu) \leq \frac{2 \rho_{T}}{\beta_{N}(\mu)}\|E(\mu)\|_{X}^{2}+\frac{2 \gamma_{N}(\mu)}{\beta_{N}(\mu)}\|E(\mu)\|_{X} .
$$

Thanks to (33), we know that $\|E(\mu)\|_{X} \leq \Delta_{N}(\mu)$, then $\frac{2 \rho_{T}}{\beta_{N}(\mu)}\|E(\mu)\|_{X} \leq \tau_{N}(\mu)$. It follows from (34), i.e.,

$$
\Delta_{N}(\mu) \leq\left[\frac{2 \gamma_{N}(\mu)}{\beta_{N}(\mu)}+\tau_{N}(\mu)\right]\left\|U(\mu)-U_{N}(\mu)\right\|_{X}
$$

6. Approximation of the eddy viscosity term. In this section we approximate the nonlinear turbulent eddy viscosity term by the EIM $[15,5]$. The EIM allows the construction an offline tensorized representation of this term that will be used in the online calculations.

Let us denote $g(\mu):=g\left(x ; \mathbf{w}_{h}(\mu)\right)=\left|\nabla \mathbf{w}_{h}(\mu)\right|(x)$. The finality of using the EIM is decoupling the $\mu$-dependence from the spatial dependence of the function $g(\mu)$, i.e.,

$$
g(\mu) \approx \mathcal{I}_{M}[g(\mu)]
$$

where we denote by $\mathcal{I}_{M}[g(\mu)]$ the empirical interpolate of $g(\mu)$. The EIM consists of constructing an RB space $W_{M}=\operatorname{span}\left\{q_{1}(\mu), \ldots, q_{M}(\mu)\right\}$, selecting these basis functions by a greedy procedure, with snapshots of $g(\mu)$. With this technique, we are able to approximate the nonlinear Smagorinsky term by a trilinear form, in the following way: $a_{S}\left(\mathbf{w}_{N} ; \mathbf{w}_{N}, \mathbf{v}_{N} ; \mu\right) \approx \hat{a}_{S}\left(\mathbf{w}_{N}, \mathbf{v}_{N} ; \mu\right)$, where

$$
\hat{a}_{S}\left(\mathbf{w}_{N}, \mathbf{v}_{N} ; \mu\right)=\sum_{k=1}^{M} \sigma_{k}(\mu) s\left(q_{k}, \mathbf{w}_{h}, \mathbf{v}_{h}\right)
$$

with $s\left(q_{k}, \mathbf{w}_{h}, \mathbf{v}_{h}\right)=\sum_{K \in \mathcal{T}_{h}} \int_{K}\left(C_{S} h_{K}\right)^{2} q_{k} \nabla \mathbf{w}: \nabla \mathbf{v} d \Omega$. 
Here $\sigma_{k}(\mu)$ for $k=1, \ldots, M$ is the solution of a lower-triangular linear system, where the second member is the value of $g\left(x ; \mathbf{w}_{h}(\mu)\right)$ in some certain points $x_{i}$. We refer to [15] for more details.

This technique allows us to linearize the eddy viscosity term. Let us recall the $\mathrm{RB}$ problem, with this last approximation of the Smagorinsky term:

$$
\left\{\begin{array}{l}
\text { Find }\left(\mathbf{u}_{N}, p_{N}\right) \in Y_{N} \times M_{N} \text { such that } \forall \mathbf{v}_{N} \in Y_{N}, \forall q_{N} \in M_{N}, \\
a\left(\mathbf{u}_{N}, \mathbf{v}_{N} ; \mu\right)+b\left(\mathbf{v}_{N}, p_{N} ; \mu\right)+\hat{a}_{S}\left(\mathbf{w}_{N}, \mathbf{v}_{N} ; \mu\right) \\
+c\left(\mathbf{u}_{D}, \mathbf{u}_{N}, \mathbf{v}_{N} ; \mu\right)+c\left(\mathbf{u}_{N}, \mathbf{u}_{D}, \mathbf{v}_{N} ; \mu\right)+c\left(\mathbf{u}_{N}, \mathbf{u}_{N}, \mathbf{v}_{N} ; \mu\right)=F\left(\mathbf{v}_{N} ; \mu\right), \\
b\left(\mathbf{u}_{N}, q_{N} ; \mu\right)=0 .
\end{array}\right.
$$

The solution $\left(\mathbf{u}_{N}(\mu), p_{N}(\mu)\right) \in X_{N}$ of (38) can be expressed as a linear combination of the basis functions:

$$
\mathbf{u}_{N}(\mu)=\sum_{j=1}^{2 N} u_{j}^{N}(\mu) \zeta_{j}^{\mathbf{v}}, \quad p_{N}(\mu)=\sum_{j=1}^{N} p_{j}^{N}(\mu) \xi_{j}^{p}
$$

Taking into account this representation, for the bilinear terms in (38), we store the parameter-independent matrices for the offline phase, as in [22], defined as

$$
\begin{array}{ll}
\left(\mathbb{A}_{N}\right)_{i j}=a\left(\zeta_{j}^{\mathbf{v}}, \zeta_{i}^{\mathbf{v}}\right),\left(\mathbb{B}_{N}\right)_{l i}=b\left(\zeta_{i}^{\mathbf{v}}, \xi_{l}^{p}\right), & i, j=1, \ldots, 2 N, l=1, \ldots, N \\
\left(\mathbb{D}_{N}\right)_{i j}=c\left(\mathbf{u}_{D}, \zeta_{j}^{\mathbf{v}}, \zeta_{i}^{\mathbf{v}}\right)+c\left(\zeta_{j}^{\mathbf{v}}, \mathbf{u}_{D}, \zeta_{i}^{\mathbf{v}}\right), & i, j=1, \ldots, 2 N
\end{array}
$$

For the convective and the Smagorinsky terms, we need to store a parameterindependent tensors of order three for the offline phase, defined as

$$
\begin{aligned}
\left(\mathbb{C}_{N}\left(\zeta_{s}^{\mathbf{v}}\right)\right)_{i j} & =c\left(\zeta_{s}^{\mathbf{v}}, \zeta_{j}^{\mathbf{v}}, \zeta_{i}^{\mathbf{v}}\right), \quad i, j, s=1, \ldots, 2 N \\
\left(\mathbb{S}_{N}\left(q_{s}\right)\right)_{i j} & =s\left(q_{s}, \zeta_{j}^{\mathbf{v}}, \zeta_{i}^{\mathbf{v}}\right), \quad i, j=1, \ldots, 2 N, s=1, \ldots, M
\end{aligned}
$$

With this tensor representation, it holds that

$$
c\left(\mathbf{u}_{N}, \zeta_{j}^{\mathbf{v}}, \zeta_{i}^{\mathbf{v}}\right)=\sum_{s=1}^{2 N} u_{s}^{N}(\mu) \mathbb{C}_{N}\left(\zeta_{s}^{\mathbf{v}}\right) \quad \text { and } \quad \hat{a}_{S}\left(\zeta_{j}^{\mathbf{v}} ; \zeta_{i}^{\mathbf{v}}\right)=\sum_{s=1}^{2 N} \sigma_{s}(\mu) \mathbb{S}_{N}\left(q_{s}\right),
$$

and thanks to that, we are able to solve problem (38), linearized by a semi-implicit evolution approach. We remark that the treatment of the approximation of the eddy viscosity term in the offline/online phase is similar to the treatment of the convective term, thanks to the tensorization done in this section.

7. Numerical results. In this section, we present some numerical tests, programmed in FreeFem ++ [17], for the reduced order Smagorinsky model [36]. We will consider two test cases: the backward-facing step problem and the lid-driven cavity problem. For both cases, we consider a range of Reynolds number for which it is known that a steady regime takes place. We obtain rates of speed-up of the computational time from several hundreds to several thousands.

7.1. Backward-facing step flow (2D). In this test, we show the numerical results of a Smagorinsky reduced order model for the backward-facing step (cf. [1]). The backward-facing step flow is laminar and reaches a steady state solution roughly up to $R e \simeq 1000$, and then it becomes transitional up to $R e \simeq 5000$. For larger values, the regime becomes turbulent (cf. [11]). The parameter that we are considering for 

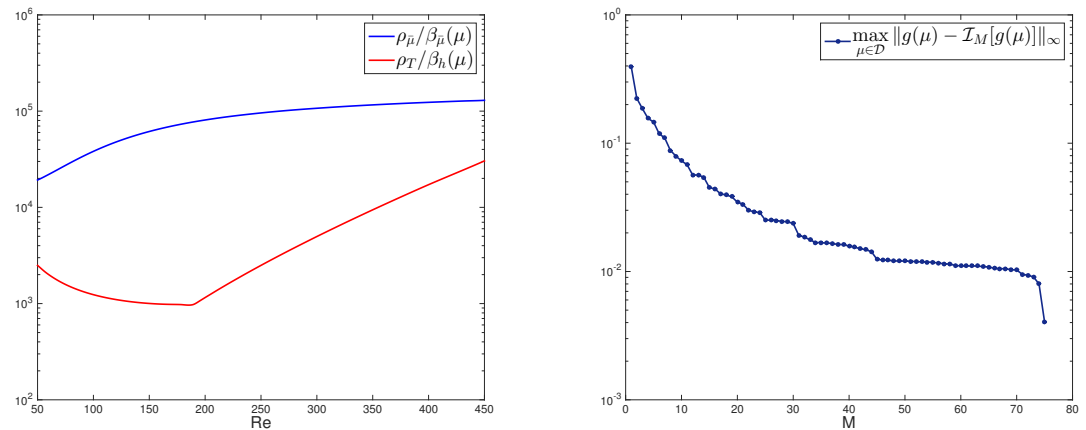

FIG. 1. Left: Comparison $\rho_{\bar{\mu}} / \beta_{\bar{\mu}}(\mu)$ and $\rho_{T} / \beta_{h}(\mu)$. Right: Convergence of the EIM algorithm.

this numerical test case is the Reynolds number with values $\mu=R e \in \mathcal{D}=[50,450]$. This means that the regime we consider in this test is fully laminar.

For the offline phase, we compute the FE approximation with the Taylor-Hood finite element, i.e, we consider $\mathbb{P} 2-\mathbb{P} 1$ for velocity-pressure. The mesh selected for this problem is composed of 10842 triangles and 5703 nodes. The FE steady state solution is computed through a semi-implicit evolution approach, and we conclude that the steady solution is reached when the relative error between two iterations is below $\varepsilon_{F E}=10^{-10}$. The numerical scheme to solve the Smagorinsky model in each time step reads as

$$
\left\{\begin{array}{l}
\text { Find }\left(\mathbf{u}_{h}^{n+1}, p_{h}^{n+1}\right) \in Y_{h} \times M_{h} \text { such that } \forall \mathbf{v}_{h} \in Y_{h}, \forall q_{h} \in M_{h} \\
\left(\frac{\mathbf{u}_{h}^{n+1}-\mathbf{u}_{h}^{n}}{\Delta t}\right)_{\Omega}+a\left(\mathbf{u}_{h}^{n+1}, \mathbf{v}_{h} ; \mu\right)+b\left(\mathbf{v}_{h}, p_{h}^{n+1} ; \mu\right)+a_{S}\left(\mathbf{w}_{h}^{n} ; \mathbf{w}_{h}^{n+1}, \mathbf{v}_{h} ; \mu\right), \\
c\left(\mathbf{u}_{h}^{n}, \mathbf{u}_{h}^{n+1}, \mathbf{v} ; \mu\right)+c\left(\mathbf{u}_{D}, \mathbf{u}_{h}^{n+1}, \mathbf{v} ; \mu\right)+c\left(\mathbf{u}_{h}^{n+1}, \mathbf{u}_{D}, \mathbf{v} ; \mu\right)=F\left(\mathbf{v}_{h} ; \mu\right), \\
b\left(\mathbf{u}_{h}^{n+1}, q_{h} ; \mu\right)=0 .
\end{array}\right.
$$

To implement the greedy algorithm, we compute beforehand the inf-sup constant $\beta_{N}(\mu),(23)$, and the Sobolev embedding constant $C_{T}$, as both appear in the a posteriori error bound estimator. Due to the fact that $U_{N}(\mu)$ is intended to be a good approximation of $U_{h}(\mu)$, in practice we use the value of $\beta_{h}(\mu)$ in place of $\beta_{N}(\mu)$. To compute the inf-sup stability factor, we consider the heuristic strategy introduced in [23]. This heuristic technique consists in interpolating the $\beta_{h}(\mu)$ map by the adaptive radial basis function algorithm (cf. [21]) for some selected values of $\mu \in \mathcal{D}$. On the other hand, to compute the Sobolev embedding constant, we use the fixed point algorithm described in [22] (section 8).

In Figure 1 (left) we compare $\rho_{\bar{\mu}} / \beta_{\bar{\mu}}(\mu)$, described in [13], and $\rho_{T} / \beta_{h}(\mu)$. These quantities are crucial for the number of basis functions necessary to ensure that $\tau_{N}(\mu)<1$. Since for our problem, $\rho_{T} / \beta_{h}(\mu)<\rho_{\bar{\mu}} / \beta_{\bar{\mu}}(\mu)$, the number of bases needed to guarantee $\tau_{N}(\mu)<1$ is lower when we use the norm $\|\cdot\|_{T}$ instead the natural norm.

To compute our RB space, we start by computing the basis functions to construct the EIM to approximate the eddy diffusion term. To evaluate the error between $g(\mu)$ and the empirical interpolation, we precompute a certain number of snapshots $\mathbf{u}_{h}(\mu)$, $\mu \in \mathcal{D}_{E I M}$. We stop the construction of the EIM bases when we reach a relative error below $\varepsilon_{E I M}=5 \cdot 10^{-3}$. This error is reached for 75 basis functions for the EIM. 

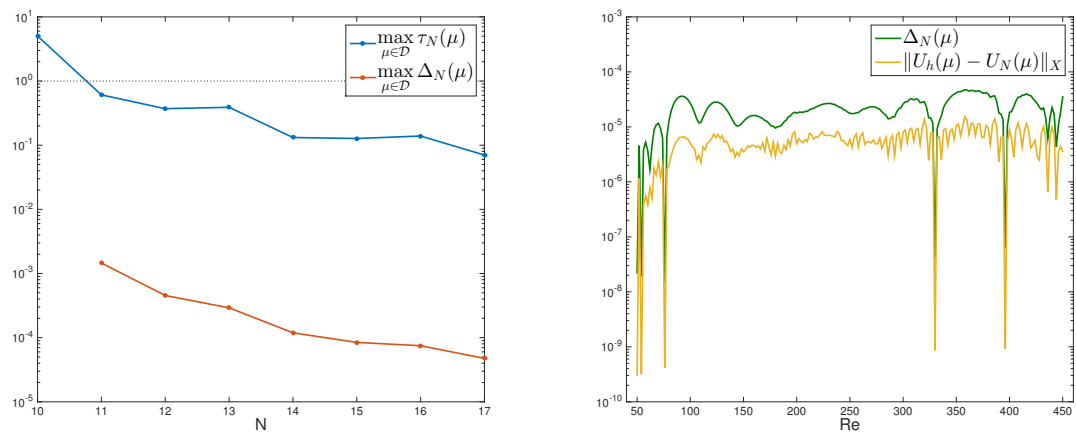

FIG. 2. Left: Convergence of the greedy algorithm. Right: Value of $\Delta_{N_{\max }(\mu)}$ and the error between the FE solution and the RB solution.

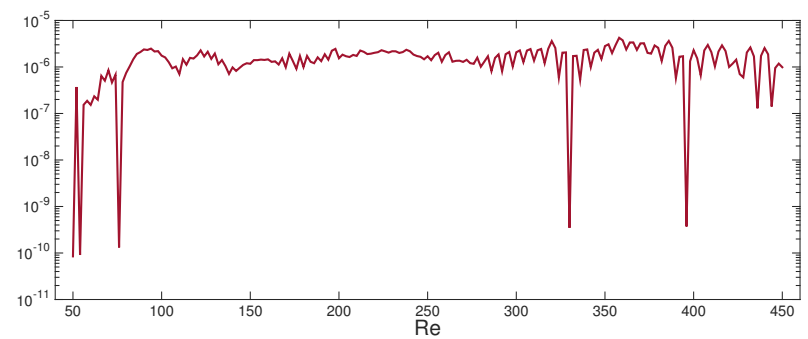

FIG. 3. Normalized error of the EIM Smagorinsky term approximation.

In Figure 1 (right), we show the evolution of the relative error, in the infinite norm, between $g(\mu)$ and its empirical interpolate.

Also, to obtain an initial guess such that $\tau_{N} \lesssim 1$, as needed by our error estimator, we first approximate the reduced manifold with some POD modes (see, e.g., [4]), and then we start our greedy algorithm. We select 10 POD modes using the snapshots computed for the EIM to start the greedy algorithm.

In Figure 2 (left), we can observe the evolution of the a posteriori error bound within the greedy algorithm. We observe that it is indeed a good error estimator, with an efficiency factor close to 10 for all $\mu \in \mathcal{D}$ (Figure 2 (right)). Due to Theorem 5.3, $\Delta_{N}(\mu)$ exists when $\tau_{N}(\mu) \leq 1$. While $\tau_{N}(\mu)>1$, we use as a posteriori error bound estimator the proper $\tau_{N}(\mu)$. We stop the greedy algorithm when we reach a tolerance of $\varepsilon_{R B}=7 \cdot 10^{-5}$, obtained when $N=N_{\max }=17$. In Figure 2 (right) we show the value of the a posteriori error bound estimator and the relative error for all $\mu \in \mathcal{D}$, at $N=N_{\max }$.

To compute the error in the EIM approximation of the Smagorinsky eddy-viscosity term, we define the following errors:

$$
e_{S}(\mu)=\sup _{\mathbf{v} \in Y}\left|a_{S}\left(\mathbf{w}_{h} ; \mathbf{w}_{h}, \mathbf{v} ; \mu\right)-a_{S}\left(\mathbf{w}_{N} ; \mathbf{w}_{N}, \mathbf{v} ; \mu\right)\right|, n_{S}(\mu)=\sup _{\mathbf{v} \in Y} a_{S}\left(\mathbf{w}_{h} ; \mathbf{w}_{h}, \mathbf{v} ; \mu\right) .
$$

In Figure 3 we show the value of $e_{S} / n_{S}$, the normalized error of the Smagorinsky term EIM approximation, for all $\mu$ in $\mathcal{D}$.

We can observe that the good approximation of the RB solution provides a good approximation for the Smagorinsky term, since the error between FE and RB solution in Figure 2 (right) and the error in Figure 3 are similar. 

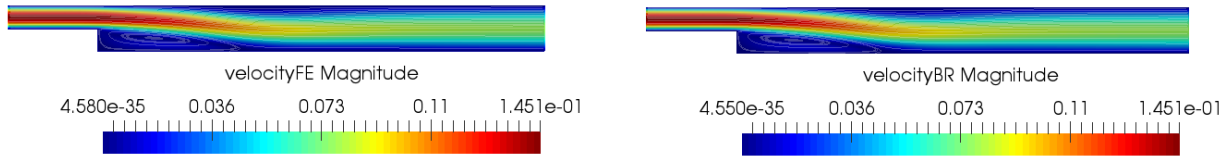

FIG. 4. FE solution (left) and RB solution (fight) for $\mu=320$.

TABLE 1

Computational time for FE solution and RB online phase, with the speedup and the relative error.

\begin{tabular}{l|ccccc}
\hline Data & $\mu=56$ & $\mu=132$ & $\mu=236$ & $\mu=320$ & $\mu=450$ \\
\hline$T_{F E}$ & $237.88 \mathrm{~s}$ & $503.57 \mathrm{~s}$ & $1055.91 \mathrm{~s}$ & $1737.74 \mathrm{~s}$ & $2948.11 \mathrm{~s}$ \\
$T_{\text {online }}$ & $1.40 \mathrm{~s}$ & $1.51 \mathrm{~s}$ & $1.69 \mathrm{~s}$ & $1.72 \mathrm{~s}$ & $2.02 \mathrm{~s}$ \\
\hline Speedup & 169 & 333 & 622 & 1008 & 1458 \\
\hline$\left\|\mathbf{u}_{h}-\mathbf{u}_{N}\right\|_{T}$ & $3.77 \cdot 10^{-7}$ & $5.33 \cdot 10^{-6}$ & $6.58 \cdot 10^{-6}$ & $1.36 \cdot 10^{-5}$ & $3.57 \cdot 10^{-6}$ \\
$\left\|p_{h}-p_{N}\right\|_{0}$ & $1.94 \cdot 10^{-8}$ & $6.97 \cdot 10^{-8}$ & $2.1 \cdot 10^{-7}$ & $4.82 \cdot 10^{-7}$ & $9.02 \cdot 10^{-8}$ \\
\hline
\end{tabular}

In Figure 4 we show a comparison between the FE velocity solution (left) and the $\mathrm{RB}$ velocity solution (right) for a chosen parameter value $\mu=320$. Note that both images are practically equal, as the error between both solutions is of order $10^{-6}$.

In Table 1, we show the results obtained for several values of $\mu$ in $\mathcal{D}$; in particular, we compare the computational time for the computation of the FE solution and the $\mathrm{RB}$ solution in the online phase. We also show the speedup rate in the computation of the RB solution, and the relative errors in $H^{1}$-norm for velocity and in $L^{2}$ for pressure. We observe a dramatic reduction of the computational time, with speedup rates over 1000 for large Reynolds number, with relative errors below the greedy tolerance. The offline phase took 3 days and 10 hours to be completed. In this time, we are considering the time to construct the RBF functions for the stability factor $\beta_{h}$, the computation of the snapshots necessaries for the EIM, and the greedy algorithm.

7.2. Lid-driven Cavity flow (2D). In this test, we apply the reduced order Smagorinsky turbulence model to the lid-driven cavity problem. We consider the nonhomogeneous Dirichlet boundary condition given by $g(x)=1$ on the lid boundary $\Gamma_{D_{g}}$ with homogeneous Dirichlet condition on $\Gamma_{D_{0}}$.

For this test, we also consider the Reynolds number as a parameter, ranging in $\mathcal{D}=[1000,5100]$. The 2D lid-driven cavity flow has a steady solution up to Reynolds 7500 (cf. [11]); thus in this range, a steady solution is well known to exist. We consider the same finite elements as the previous problem (Taylor-Hood), and we use a regular mesh with 5000 triangles and 2601 nodes.

In Figure 5 (left), we show the comparison between $\rho_{\bar{\mu}} / \beta_{\bar{\mu}}(\mu)$ and $\rho_{T} / \beta_{h}(\mu)$ as in section 7.1. Again, the number of basis functions needed to guarantee that $\tau_{N}(\mu)<1$ in this problem is lower if we chose the norm $\|\cdot\|_{T}$ instead of choosing the natural norm. In this numerical test, we need $M_{\max }=22$ basis functions in the EIM algorithm until reaching the tolerance for the relative error of $\varepsilon_{E I M}=5 \cdot 10^{-4}$. In Figure 5 (right), we show the convergence of the EIM algorithm.

For the greedy algorithm, we prescribe a tolerance of $\varepsilon_{R B}=5 \cdot 10^{-5}$. This tolerance is reached for $N_{\max }=12$ basis functions. Note that, in this case, $N=8$ basis functions are needed in order to ensure that $\tau_{N}(\mu)<1$ for all $\mu$ in $\mathcal{D}$. In Figure 6 (left) we show the convergence of the greedy algorithm, and in Figure 6 (right) we show the value of the error and the a posteriori error bound for all $\mu$ in $\mathcal{D}$. 

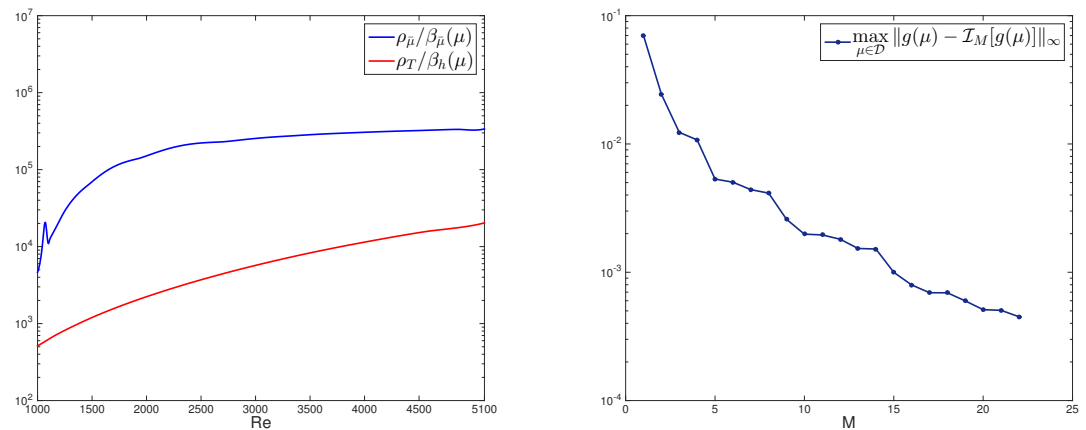

FIG. 5. Left: Comparison $\rho_{\bar{\mu}} / \beta_{\bar{\mu}}(\mu)$ and $\rho_{T} / \beta_{h}(\mu)$. Right: Convergence of the EIM algorithm.
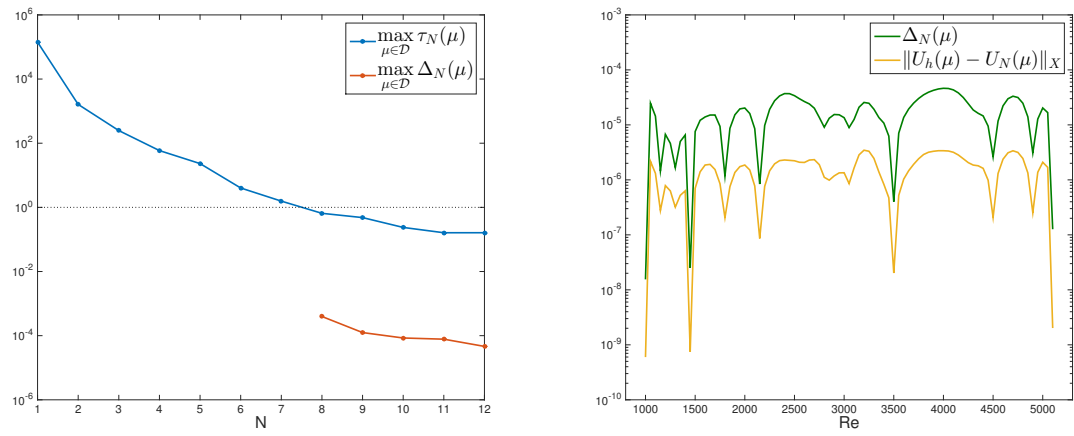

FIG. 6. Left: Convergence of the greedy algorithm. Right: Value of $\Delta_{N_{\max }}(\mu)$ and the error between the FE solution and the RB solution.

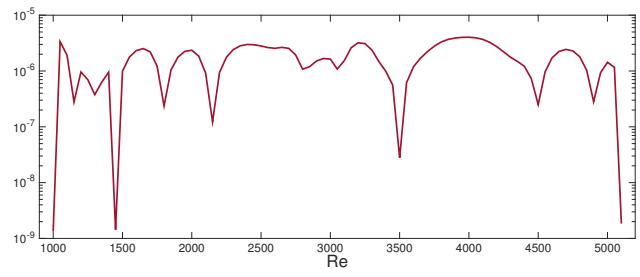

FIG. 7. Normalized error of the EIM Smagorinsky term approximation.

As in section 7.1, we compute the error $e_{S}(\mu) / n_{S}(\mu)$, in order to compute the error in the EIM approximation of the Smagorinsky term. In Figure 7, we show this error for this numerical test. Again, a good approximation between FE and RB solution provides a good EIM approximation of the Smagorinsky term. In Figure 8 we show a comparison between the $\mathrm{FE}$ velocity solution and the $\mathrm{RB}$ velocity solution for a chosen parameter value $\mu=4521$. Again, both images are practically equal, as the error between both solutions is of order $10^{-7}$.

Finally, we show in Table 2 a summary of the results obtained for several values of $\mu$ in $\mathcal{D}$. For this test, we also observe a dramatic speedup in the computation of the numerical solution, even larger than in the backward-facing step test. These large speed-up factors are possibly due to the high turbulent levels of viscosity introduced by the Smagorinsky turbulence model. The offline phase of this test took 2 days to be completed.

Copyright $@$ by SIAM. Unauthorized reproduction of this article is prohibited. 

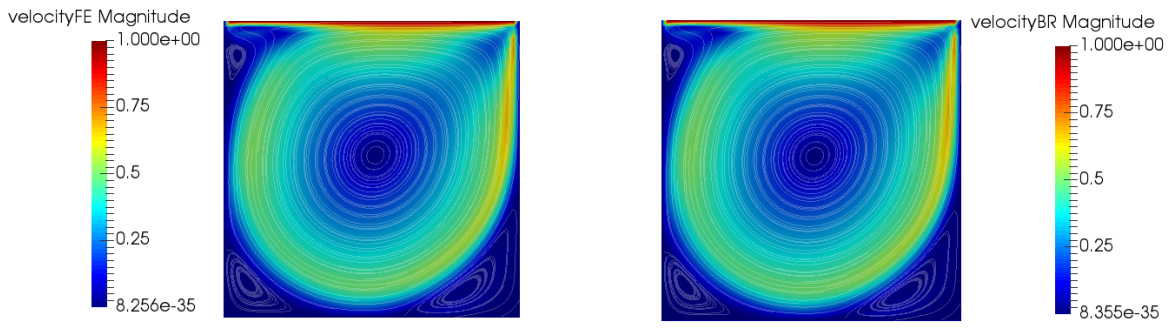

FIG. 8. FE solution and RB solution for $\mu=4521$.

TABLE 2

Computational time for $F E$ solution and $R B$ online phase, with the speedup and the relative error.

\begin{tabular}{l|ccccc}
\hline Data & $\mu=1610$ & $\mu=2751$ & $\mu=3886$ & $\mu=4521$ & $\mu=5100$ \\
\hline$T_{F E}$ & $638.02 \mathrm{~s}$ & $1027.62 \mathrm{~s}$ & $1369.49 \mathrm{~s}$ & $1583.08 \mathrm{~s}$ & $1699.52 \mathrm{~s}$ \\
$T_{\text {online }}$ & $0.47 \mathrm{~s}$ & $0.47 \mathrm{~s}$ & $0.47 \mathrm{~s}$ & $0.49 \mathrm{~s}$ & $0.52 \mathrm{~s}$ \\
\hline Speedup & 1349 & 2182 & 2899 & 3243 & 3227 \\
\hline$\left\|\mathbf{u}_{h}-\mathbf{u}_{N}\right\|_{T}$ & $1.91 \cdot 10^{-6}$ & $1.87 \cdot 10^{-6}$ & $3.28 \cdot 10^{-6}$ & $6.26 \cdot 10^{-7}$ & $3.17 \cdot 10^{-9}$ \\
$\left\|p_{h}-p_{N}\right\|_{0}$ & $1.18 \cdot 10^{-7}$ & $3.65 \cdot 10^{-7}$ & $3.78 \cdot 10^{-7}$ & $8.34 \cdot 10^{-8}$ & $1.88 \cdot 10^{-9}$ \\
\hline
\end{tabular}

8. Conclusions. In this paper we have developed an RB Smagorinsky model, using the EIM to linearize the nonlinear eddy viscosity of the Smagorinsky model.

We have developed an a posteriori error bound estimator for the Smagorinsky model, extending the theory in the literature for the incompressible Navier-Stokes equations (e.g., $[22,13])$. With the a posteriori error bound estimator we can compute the offline phase in a efficient way, including the computation of the inf-sup stability interpolator, that provides a fast reliable approximation of the inf-sup stability factor value for each parameter value.

We have presented numerical results for two benchmark cases, where we have shown the accuracy of our reduced model and the dramatic reduction of the computational time for both cases, which is typically divided by several thousands. This high speed-up rate is possibly due to the high dissipative effect of the Smagorinsky turbulence model. Extensions to less dissipative turbulence models of VMS kind are in progress.

Acknowledgments. We acknowledge Professor Yvon Maday (LJLL) for his support and guidelines during this research.

\section{REFERENCES}

[1] B. Armali, F. Durst, J. Pereira, And B. Schönung, Experimental and theoretical investigation of backward-facing step flow, J. Fluid Mech., 127 (1983), pp. 473-496.

[2] J. BAIges, R. CoDina, AND S. IDELSOHN, Explicit reduced-order models for the stabilized finite element approximation of the incompressible Navier-Stokes equations, Internat. J. Numer. Methods Fluids, 72 (2013), pp. 1219-1243.

[3] M. J. Balajewicz, E. H. Dowell, and B. R. Noack, A novel model order reduction approach for Navier-Stokes equations at high Reynolds number, J. Fluid Mech., 729 (2013), pp. 285308.

[4] F. Ballarin, A. Manzoni, A. Quarteroni, And G. Rozza, Supremizer stabilization of PODGalerkin approximation of parametrized steady incompressible Navier-Stokes equations, Internat. J. Numer. Methods Engrg., (2014), pp. 1136-1161.

[5] M. Barrault, Y. Maday, N. C. Nguyen, and A. T. Patera, An empirical interpolation method: Application to efficient reduced-basis discretization of partial differential equations, C. R. Acad. Sci. Paris Sér. I Math., 339 (2004), pp. 667-672. 
[6] C. Bernardi, Y. Maday, and F. Rapetti, Discrétisations variationelles de Problémes aux limites elliptiques, Math. Appl., 45, 2004.

[7] F. BREzZI, On the existence, uniqueness, and approximation of saddle point problems arising from Lagrangian multipliers., RAIRO Anal. Numér., 2 (1974), pp. 129-151.

[8] F. BREzZI, J. RAPpaz, AND P. RAVIART, Finite dimensional approximation of nonlinear problems, Numer. Math., 36 (1980), pp. 1-25.

[9] G. Caloz and J. Rappaz, Numerical analysis for nonlinear and bifurcation problems, Handb. Numer. Anal., 51 (1997), pp. 487-637.

[10] T. Chacon, An analysis technique for stabilized finite element solution of incompressible flows, ESAIM Math. Model. Numer. Anal., 35 (2001), pp. 57-89.

[11] T. Chacon And R. Lewandowski, Mathematical and Numerical Foundations of Turbulence Models and Applications, Springer, New York, 2014.

[12] M. Couplet, P. Sagaut, And C. Basdevant, Intermodal energy transfers in a proper orthogonal decomposition-Galerkin representation of a turbulent separated flow, J. Fluid Mech., 491 (2003), pp. 257-284.

[13] S. DePARIS, Reduced basis error bound computation of parameter-dependent Navier-Stokes equations by the natural norm approach, SIAM J. Sci. Comput., 46 (2008), pp. 2039-2067.

[14] S. Deparis And G. Rozza, Reduced basis method for multi-parameter-dependent steady NavierStokes equations: Applications to natural convection in a cavity, J. Comput. Phys., 228 (2009), pp. 4359-4378.

[15] M. A. Grepl, Y. Maday, N. C. Nguyen, and A. T. Patera, Efficient reduced-basis treatment of nonaffine and nonlinear partial differential equations, ESAIM Math. Model. Numer. Anal., 41 (2007), pp. 575-605.

[16] M. Gunzburger, N. Jiang, And M. Schneier, An Ensemble-Proper Orthogonal Decomposition Method for the Nonstationary Navier-Stokes Equations, https://arxiv.org/abs/1603. 04777, 2016.

[17] F. Несht, New development in FreeFem++, J. Numer. Math., 20 (2012), pp. 251-265.

[18] H. Herrero, Y. Maday, and F. Pla, RB (Reduced basis) for RB (Rayleigh-Bnard), Comput. Methods Appl. Mech. Engrg., 261-262 (2013), pp. 132-141.

[19] J. S. Hesthaven, G. Rozza, and B. Stamm, Certified Reduced Basis Methods for Parametrized Partial Differential Equations, Springer, New York, 2015.

[20] P. Holmes, J. L. Lumley, and G. Berkooz, Turbulence, Coherent Structures, Dynamical Systems and Symmetry, Camnridge University Press, Cambridge, 1996.

[21] T. J. MackMan and C. B. Allen, Investigation of an adaptive sampling method for data interpolation using radial basis functions, Internat. J. Numer. Methods Engrg., 83 (2010), p. 915-938.

[22] A. MAnzoni, An efficient computational framework for reduced basis approximation and a posteriori error estimation of parametrized Navier-Stokes flows, ESAIM Math. Model. Numer. Anal., 48 (2014), pp. 1199-1226.

[23] A. Manzoni And F. Negri, Rigorous and heuristic strategies for the approximation of stability factors in nonlinear parametrized PDEs., Adv. Comput. Math., 41 (2015), pp. 1255-1288.

[24] F. Negri, A. Manzoni, AND G. RozzA, Reduced basis approximation of parametrized optimal flow control problems for the Stokes equations, Comput. Math. Appl., 69 (2015), pp. 319336.

[25] G. Pitton, A. Quaini, And G. Rozza, Computational reduction strategies for the detection of steady bifurcations in incompressible fluid-dynamics: Applications to Coanda effect in cardiology, J. Comput. Phys., 344 (2017), pp. 534-557.

[26] G. Pitton and G. Rozza, On the application of reduced basis methods to bifurcation problems in incompressible fluid dynamics, J. Sci. Comput., 73 (2017), pp. 157-177.

[27] C. Prudhomme, D. V. Rovas, K. Veroy, L. Machiels, Y. Maday, and A. T. Patera, Reliable real-time solution of parametrized partial differential equations: Reduced-basis output bound methods, J. Fluids Eng., 124 (2002), pp. 70-80.

[28] A. Quarteroni, A. Manzoni, And F. Negri, Reduced Basis Methods for Partial Differential Equations: An Introduction, Springer, New York, 2015.

[29] A. Quarteroni, A. Manzoni, and G. Rozza, Certified reduced basis approximation for parametrized partial differential equations and applications, J. Math. Ind., 1 (2011).

[30] G. Rozza, D. B. P. Huynh, And A. T. Patera, Reduced basis approximation and a posteriori error estimation for affinely parametrized elliptic coercive partial differential equations, Arch. Comput. Methods Eng., 15 (2008), pp. 229-275.

[31] G. Rozza, D. P. Huynh, And A. Manzoni, Reduced basis approximation and a posteriori error estimation for Stokes flows in parametrized geometries: Roles of the inf-sup stability constant, Numer. Math., (2013), pp. 115-152.

Copyright $@$ by SIAM. Unauthorized reproduction of this article is prohibited. 
[32] G. Rozza And K. Veroy, On the stability of the reduced basis method for Stokes equations in parametrized domains, Comput. Methods Appl. Mech. Engrg., 196 (2007), pp. 1244-1260.

[33] S. Rubino, Numerical Modeling of Turbulence by Richardson Number-Based and VMS Models, Ph.D. thesis, University of Seville, 2014.

[34] P. Sagaut, Large Eddy simulation for incompressible flows: An introduction, Springer, New York, 2001.

[35] L. Sirovich, Turbulence and the dynamics of coherent structures. Parts I-III, Quart. Appl. Math., 45 (1987), pp. 561-590.

[36] J. Smagorinsky, General circulation experiments with the primitive equations. I. The basic experiment., Monthly Weather Rev., 91 (1963), pp. 99-164.

[37] K. Veroy and A. T. Patera, Certified real-time solution of the parametrized steady incompressible Navier-Stokes equations: Rigorous reduced-basis a posteriori error bounds, Internat. J. Numer. Methods Fluids, 47 (2005), pp. 773-788.

[38] Z. Wang, I. Akhtar, J. Broggand, and T. Iliescu, Proper orthogonal decomposition closure models for turbulent flows: A numerical comparison, Comput. Methods Appl. Mech. Engrg., 237-240 (2012), pp. 10-26.

[39] Z. WANG AND T. ILIESCU, Variational multiscale proper orthogonal decomposition: NavierStokes equations, Numer. Methods Partial Differential Equations, 30 (2014), pp. 641-663.

[40] M. Yano And A. T. Patera, A space-time variational approach to hydrodynamic stability theory, Proc. Roy. Soc. A, 469 (2013).

Copyright (c) by SIAM. Unauthorized reproduction of this article is prohibited. 